Ultramicroscopy. 2014 October ; 145: 36-49. doi:10.1016/j.ultramic.2014.01.007.

\title{
A Novel Low Energy Electron Microscope for DNA Sequencing and Surface Analysis
}

\author{
M. Mankos ${ }^{a,+}$, K. Shadman ${ }^{a}$, H.H.J. Persson ${ }^{b}$, A.T. N'Diaye ${ }^{a, c}$, A.K. Schmid ${ }^{c}$, and R.W. \\ Davis $^{b}$ \\ aElectron Optica Inc., 1000 Elwell Court \#110, Palo Alto, California 94303 \\ bStanford Genome Technology Center, Stanford University School of Medicine, 855 California \\ Avenue, Palo Alto, CA 94304 \\ 'NCEM, Lawrence Berkeley National Laboratory, 1 Cyclotron Road, Berkeley, CA 94720, USA
}

\section{Abstract}

Monochromatic, aberration-corrected, dual-beam low energy electron microscopy (MAD-LEEM) is a novel technique that is directed towards imaging nanostructures and surfaces with subnanometer resolution. The technique combines a monochromator, a mirror aberration corrector, an energy filter, and dual beam illumination in a single instrument. The monochromator reduces the energy spread of the illuminating electron beam, which significantly improves spectroscopic and spatial resolution. Simulation results predict that the novel aberration corrector design will eliminate the second rank chromatic and third and fifth order spherical aberrations, thereby improving the resolution into the sub-nanometer regime at landing energies as low as one hundred electron-Volts. The energy filter produces a beam that can extract detailed information about the chemical composition and local electronic states of non-periodic objects such as nanoparticles, interfaces, defects, and macromolecules. The dual flood illumination eliminates charging effects that are generated when a conventional LEEM is used to image insulating specimens. A potential application for MAD-LEEM is in DNA sequencing, which requires high resolution to distinguish the individual bases and high speed to reduce the cost. The MAD-LEEM approach images the DNA with low electron impact energies, which provides nucleobase contrast mechanisms without organometallic labels. Furthermore, the micron-size field of view when combined with imaging on the fly provides long read lengths, thereby reducing the demand on assembling the sequence. Experimental results from bulk specimens with immobilized single-base oligonucleotides demonstrate that base specific contrast is available with reflected, photo-emitted, and Auger electrons. Image contrast simulations of model rectangular features mimicking the individual nucleotides in a DNA strand have been developed to translate measurements of contrast on bulk DNA to the detectability of individual DNA bases in a sequence.

(C) 2014 Elsevier B.V. All rights reserved.

${ }^{+}$Corresponding author, electronic mail: marian@electronoptica.com.

Publisher's Disclaimer: This is a PDF file of an unedited manuscript that has been accepted for publication. As a service to our customers we are providing this early version of the manuscript. The manuscript will undergo copyediting, typesetting, and review of the resulting proof before it is published in its final citable form. Please note that during the production process errors may be discovered which could affect the content, and all legal disclaimers that apply to the journal pertain. 


\section{Keywords}

Low energy electron microscopy; Monochromator; Aberration correction; Dual beam illumination; Energy filtering; DNA Sequencing; Contrast

\section{Introduction}

Significant demand exists for the development of novel techniques capable of imaging nanostructures, macromolecules, and surfaces to provide analytical capabilities with subnanometer resolution. Many specimens of interest, including biological macromolecules, sub-cellular structures, and protein crystals are difficult to image in standard (scanning) transmission electron microscopes ((S)TEM) as well as in scanning electron microscopes (SEM) because of a lack of contrast, radiation damage, and charging effects induced by the illuminating electron beam. Recently, more 'gentle' low-voltage (S)TEMs have been developed that reduce the beam energy to $20 \mathrm{keV}[1,2]$. However, the application of these instruments becomes limited to extremely thin samples, while the damage to biological specimens remains significant. Electron landing energies have been reduced to $1 \mathrm{keV}$ or less in low-voltage SEMs; however the lack of resolution and the resulting, shallow deposition of charge on the sample enhances charge induced beam distortion and blur, which limits the utility of these microscopes. Environmental SEMs [3] mitigate charging effects by introducing gas at relatively high pressures; however, the scattering of the electron beam by the gas molecules degrades the resolution to inadequate values, particularly at low beam energies.

Low energy electron microscopy (LEEM) is a technique that was developed in the 1980's by professor E. Bauer's group [4]. Similar to a SEM, a LEEM detects electrons emitted, scattered, or reflected from the surface of a specimen. However, rather than scanning a finely focused beam across the specimen, the microscope illuminates the sample surface with a broad beam of electrons, like a TEM. The illumination and projection optics are separated by a beam separator, which is a magnetic prism array that deflects the electron beam towards the axis of the objective lens. The objective lens is an immersion cathode lens, which decelerates the electrons from the column transport energy, typically of order 10-25 $\mathrm{keV}$, to a landing energy ranging from $0 \mathrm{eV}$ to a few $100 \mathrm{eV}$. In the opposite direction, moving away from the specimen, the objective lens simultaneously accelerates the reflected and emitted electrons and forms an image that is magnified by the projection optics on a scintillating screen.

The extremely low energy of the illuminating electrons makes LEEM an exquisitely sensitive surface imaging technique, capable of imaging single atomic layers with high contrast [5]. Furthermore, the low electron impact energies prevent radiation damage to delicate samples such as biological molecules. The main drawbacks of LEEM are its increased susceptibility to chromatic aberrations and charging effects. In spite of the short deBroglie wavelength, which is in the range of 4 Angstroms for $10 \mathrm{eV}$ electrons, the lateral resolution of conventional LEEM instruments is limited to a few nanometers. In addition, 
when a conventional LEEM is used to image insulating specimens, sample charging adversely impacts the low energy electron beam, blurring and distorting the image.

This paper describes the results to date of a phase I study for sequencing DNA with a MADLEEM instrument. This work has been focused on the simulation of the achievable spatial resolution of this microscope as well as on the experimental characterization of nucleotidespecific contrast of bulk homo-polymers. The ultimate goal of this work is to produce a microscope that is capable of imaging label-free bases in single- and double-stranded DNA sequences of practically unlimited length, with sub-nanometer resolution, at an affordable cost, and with no radiation damage. To this end, the second phase will be devoted to building a MAD-LEEM prototype, preparing substrates with linearized DNA, and validating the resolving power of the instrument by imaging selected sequences of DNA.

MAD-LEEM is a novel instrument that aims to overcome the aforementioned drawbacks of current LEEMs. The instrument utilizes an energy filtering mechanism to reduce the energy spread of the electrons scattered (or emitted) by the sample to a few tens of meV. The same mechanism is also used by the monochromator to reduce the energy spread of the illuminating electrons. It also employs electron mirrors as aberration correcting elements to achieve sub-nanometer resolution over a relatively large field of view. Last, it illuminates the sample with a second low energy, overlapping electron beam with a tunable landing energy to neutralize the charge deposited by the imaging beam, thereby eliminating surface charging.

\section{MAD-LEEM Electron Optics}

Figure 1 shows a schematic layout of the MAD-LEEM electron-optical column, containing two independent illumination beams, a monochromator, an aberration corrector, and an energy filter, which are integrated into a single column by three beam separators. The basic features of MADLEEM and its simulated performance have been reported $[8,16]$. The key new features of this electron-optical design are the pentode mirror aberration corrector, the knife-edge based imaging energy filter, the addition of the photoemission electron microscopy (PEEM) imaging mode and the modified and improved objective lens. Detailed simulations of the system performance based on these new features have been undertaken showing significant improvements.

The beam separators are based on compact, double-focusing magnetic prism arrays composed of uniform magnetic fields of different strengths and lengths. Each separator quadrant deflects the beam by 90 degrees and transfers stigmatically two planes, the diffraction (slit) and (achromatic) image planes, with unit magnification. The excitations of the coils are chosen so that the prism behaves as a thick, round field lens along the curved axis. The contribution of the individual beam separators to the overall aberration of the system is minimized by placing a highly-magnified image at the achromatic plane, at the center of this field lens.

The monochromator and energy filter are needed to reduce the energy spread of the beam to $100 \mathrm{meV}$ or less, which is a prerequisite for obtaining detailed information about the chemical composition, interatomic bonding, and local electronic states of macromolecules 
[6]. The aberration corrector utilizes an electron mirror that can compensate one or more aberrations of the objective lens. The dual beam approach eliminates specimen charging by illuminating the sample specimen with two superimposed flood beams, where the landing energy of the secondary beam is tuned to remove the charge deposited by the first [7].

\subsection{Illumination optics}

The illumination configuration shown in Fig. 1a has two branches, one for the higher energy imaging beam (solid lines) and one for the mirror, charge balance beam (dash lines). Both beams are recombined by the main beam separator.

2.1.1 Monochromator-The MAD-LEEM illumination optics includes a monochromator for the imaging beam that can reduce the electron energy spread of commonly used electron sources (e.g. thermionic and thermally assisted field emitters) from the range of 0.5 to $2 \mathrm{eV}$ to less than a few tens of meV. It utilizes the combination of a second energy-dispersive beam separator and an electron mirror $[8,9]$ to filter electrons with energies that are lower or higher than the selected nominal energy, $\mathrm{E}_{0}$. The electron source image is transferred via the slit planes of the main and second beam separators onto the objective lens back-focal plane, while its angular distribution is transferred via the beam separator achromatic planes and used to illuminate the specimen. The practical realization of the monochromator incorporates several transfer lenses with net zero rotation and an electron mirror to account for the residual uncertainties in the optical parameters of the separator and mirror [8]. This arrangement uses a knife edge as the energy-selecting device, a much simpler and more reliable design when compared to the narrow, often sub-micrometer slits needed in typical monochromator applications. Furthermore, the symmetry introduced by the double pass through the monochromator eliminates the energy dispersion of the beam separator.

The effect of the reduction in energy spread of the incident electron beam to a few tens of $\mathrm{meV}$ is twofold: first, it can improve the energy resolution in spectroscopic imaging modes, and second, it reduces the higher rank chromatic aberrations remaining after the correction of the primary chromatic aberration, in particular at low landing energies. The impact of the monochromator is less noticeable at higher electron energies as the relative contribution of chromatic aberrations is reduced.

2.1.2 The charge balance beam-After exiting the monochromator, the nearly monochromatic imaging beam passes through transfer lenses and an Einzel lens, a threeelectrode electrostatic lens with both outer electrodes at the ground potential and with the central electrode biased at a high negative potential. The beam passes through this Einzel lens and enters the main beam separator, which deflects the beam into a third beam separator. This separator deflects the beam by another 90 degrees into the objective lens and, in the process, cancels the dispersion introduced by the main beam separator.

The charge balance beam is generated by a second electron gun, biased to a potential that is a few hundred volts more positive than the electron gun that generates the imaging beam. The charge balance beam is collimated by the illumination optics into the main beam separator, which deflects the beam by 90 degrees towards the Einzel lens. The central electrode of the Einzel lens is biased slightly more negative than the charge balance electron 
gun to reflect the charge balance beam back towards the main beam separator, which deflects it towards the third beam separator. Compared to the imaging beam, the charge balance beam has a lower kinetic energy when passing through the main beam separator. Consequently, it is deflected by slightly more than 90 degrees. A transfer lens located midway between the main and the third beam separator focuses the charge balance beam emanating from the achromatic plane of the main beam separator at the achromatic plane of the third beam separator. This lens makes the imaging and the charge balance beam coaxial after the 90 degree deflection by the third beam separator.

In LEEM mode, the imaging beam is characterized by a lower landing energy. In other words, the role of the imaging and the charge balance beams is interchanged [7]. Here, the imaging beam deposits a net number of electrons on the sample, and the landing energy of the charge balance beam must be tuned to produce a secondary yield greater than 1.0. The typical difference in the landing energies of the two beams is $300 \mathrm{eV}$. This difference may be adjusted along with the relative ratios of the two beam currents to neutralize the surface.

After the deflection by the main beam separator and the focusing by the transfer lens, both beams are deflected by the third beam separator into the cathode objective lens. The objective lens decelerates both beams to form two low-energy, parallel flood beams illuminating the specimen.

\subsection{Projection Optics}

The electrons reflected and emitted by the specimen are accelerated away from the specimen and focused by the objective lens to form a magnified, two-dimensional image blurred by the aberrations of the objective lens. The returning electron beam enters the third beam separator and is deflected towards a symmetry electron mirror. The strength of the third beam separator is adjusted so that electrons with nominal energy $\mathrm{E}_{0}$ (solid lines) are deflected by 90 degrees and enter along the axis of the symmetry electron mirror, while electrons with slightly lower $\left(\mathrm{E}_{0}-\delta \mathrm{E}_{0}\right)$ or higher $\left(\mathrm{E}_{0}+\delta \mathrm{E}_{0}\right)$ energy are deflected by a slightly larger or smaller angle, respectively, as a result of the energy dispersion of the beam separator. The axial bundle of electron rays with energies in the range $\left(\mathrm{E}_{0}-\delta \mathrm{E}_{0}, \mathrm{E}_{0}+\delta \mathrm{E}_{0}\right)$ thus appears to emanate from a point near the center plane of the third beam separator, its achromatic plane. Electrons emerging from this achromatic plane are focused at the reflection surface of the symmetry electron mirror, which is biased more negative than the specimen by a few hundred or thousand Volts to reflect the electrons back towards the third beam separator. The electron rays are then refocused by the transfer lens at the achromatic plane of the third magnetic beam separator and deflected towards another transfer lens, which focuses the beam into a mirror aberration corrector (MAC) that corrects the spherical and chromatic aberrations of the objective lens.

2.2.1 The energy filter-The symmetry electron mirror can also be utilized for energy filtering. A knife edge introduced in front of the symmetry mirror in combination with the dispersion of the third beam separator selects electrons with nominal energy $\mathrm{E}_{0}$ for imaging and thus operates as an energy filter in a fashion similar to the monochromator described above [10]. The deflection strength of the third beam separator can be tuned to select a 
nominal energy $\mathrm{E}_{0}$ in the range of $0 \mathrm{eV}$ (charge balance electrons) to the electron landing energy (elastically backscattered electrons). The energy band-pass of the final filtered image can be selected by adjusting the position of the knife edge aperture. Utilizing this design, an energy bandwidth of a few tens of meV should be achievable.

2.2.2 The mirror aberration corrector (MAC)—The second pass through the third beam separator eliminates the energy dispersion introduced into the beam prior to being mirrored. Consequently, the beam enters the MAC dispersion free, which eliminates aberrations that would arise from the combination of dispersion with the chromatic and spherical aberrations of the electron mirror. These combination aberrations not only could dominate the aberrations of the objective lens, but more to the point, cannot be corrected by the MAC. The specimen image is then transferred from the center of the third beam separator onto the object plane of the MAC. The MAC images the object onto itself, without forming an intermediate image in the MAC. A field lens is placed at the image plane to focus the diffraction/slit plane and control the field rays. The MAC is then tuned to cancel the combined aberrations of the objective lens, as well as the aberrations from the intermediate transfer and field lenses. The aberration-corrected beam is refocused at the achromatic plane of the third beam separator, which deflects the beam towards the main beam separator. The 90 degree deflection introduces dispersion into the beam. Another transfer lens located halfway between the third and the main beam separator focuses the beam, which appears to emanate from the achromatic plane of the third beam separator, onto the achromatic plane of the main beam separator. The transfer lens ensures that the dispersion that was introduced is eliminated by the 90 degree deflection in the main beam separator- a desirable effect for minimizing aberrations in the projection optics. After the final deflection by the main beam separator, the aberration-corrected imaging beam is transported into the projection optics and magnified on a viewing screen.

2.2.3 The (X)PEEM mode of operation-As with most of the LEEM instruments, the MAD-LEEM optics can be readily operated in X-ray or photo-emitted electron modes by illuminating the specimen with an X-ray or a UV source. However, when compared to conventional PEEM instruments, the MAD-LEEM offers several advantages: the aberration corrector removes the spherical and chromatic aberrations that deteriorate the spatial resolution of conventional PEEM instruments; the energy filter allows for energy-selective imaging; and the charge balance beam enables the imaging of insulating specimens, which otherwise would charge under UV or X-ray illumination.

The diagram in Fig. 1b describes the implementation of photoemission electron microscopy utilizing the MAD-LEEM optics. In this imaging mode, a flood beam of photons generated by the X-ray or UV source illuminates the specimen and generates a beam of photo-emitted electrons (solid line) with a range of kinetic energies. The photo-emitted electrons are accelerated by the objective lens to form a magnified image blurred by aberrations. The charge balance beam (dashed lines) is used to mitigate charging effects caused by the X-ray or UV illumination. The remainder of the optics works in the same fashion as in the LEEM mode discussed above. The (X)PEEM mode can be a standalone, simplified, version of MADLEEM for dedicated applications. 
The energy filter described above can be used to select electrons from a small energy window with which to form the image in order to deliver analytical information about the specimen such as its elemental composition. The application of the aperture to the wide angular distribution of the photo-emitted electrons combined with the additional filtering by the energy window can significantly reduce the fraction of the electrons that reach the detection plane when compared to the collection efficiency of the reflected electrons in the LEEM imaging mode. However the resulting loss in the intensity is partially recovered by the greater nominal contrast of the X-ray signal and by the introduction of the MAC, which significantly increases the optimum aperture angle as well as the tolerable energy spread.

It should be noted that the layout shown in Fig. 1 represents the conceptual design of the MAD-LEEM column and includes only the critical electron-optical elements needed to illustrate the imaging principles. Many of the elements typically present in a detailed column design such as transfer and field lenses and alignment and stigmation coils are omitted here for clarity. In summary, the source is imaged onto the beam separator slit planes and subsequently into the objective lens back-focal plane, while the source angular distribution is imaged onto the beam separator achromatic planes, which is projected to illuminate the specimen surface. On the imaging side, a highly magnified image of the sample surface is placed at the center, achromatic planes of the beam separators, while the diffraction pattern is placed at the energy-dispersed separator slit planes.

\subsection{Simulated Predictions for Resolution Performance}

The best spatial resolution that is achieved in practice in a LEEM without aberration correction is between 4 and $5 \mathrm{~nm}$. At very low electron energies, the resolution is limited by diffraction because of the inverse square root relationship between wavelength and energy: at $1 \mathrm{eV}$, for example, the electron deBroglie wavelength is $1.2 \mathrm{~nm}$. At an electron energy of $150 \mathrm{eV}$, the wavelength falls to $1 \AA$, and chromatic and spherical aberrations limit the achievable resolution. Although the monochromator can reduce the chromatic aberration significantly, the reduction in the energy spread is not sufficient to improve the resolution to values below $1 \mathrm{~nm}[6,11]$.

In a LEEM equipped with an aberration corrector, the spatial resolution can be further improved by eliminating one or more aberrations of the objective lens. Conventional rotationally symmetric electron lenses focus more strongly electron rays with larger entrance slopes and lower energies, resulting in positive spherical and negative chromatic aberration coefficients. Electron mirrors, on the other hand, can be adjusted to focus more weakly the aforementioned rays, thus yielding spherical and chromatic aberration coefficients with opposite signs. Schmidt [12] and Tromp [13] utilized a tetrode MAC [14] that eliminated both the chromatic and spherical aberration of the objective lens and experimentally improved the resolution to about $2 \mathrm{~nm}$. With the primary spherical and chromatic aberrations eliminated by the MAC, the resolution is then determined by diffraction and the remaining higher rank chromatic and $5^{\text {th }}$ order geometric aberrations.

The electron-optical properties of the objective lens are key to understanding the resolution limit attainable in a LEEM. The specimen is biased at a high negative voltage, typically between 10 and $30 \mathrm{kV}$, and thus immersed in a high electrostatic field. This field when 
combined with the focusing effect of the objective lens produces a magnified image of the specimen surface. The geometry of a magnetic objective lens adopted for our instrument, which is optimized for imaging low energies commonly used in LEEM/PEEMs, is shown in Fig. 2. In comparison to the previous design [8], the geometry of the magnetic pole pieces was modified significantly to reduce the objective lens aberrations. In addition, the modified objective lens design allows for a much more compact layout as the first image plane is now at $130 \mathrm{~mm}$ (compared to $260 \mathrm{~mm}$ in ref. [8]). The objective lens has an accelerating field of approximately $5 \mathrm{kV} / \mathrm{mm}$ at the specimen surface and produces an electron beam with an energy of $20 \mathrm{keV}$. Higher electric fields improve the resolution; however, they also present a risk for arcing. The geometry of the electrodes and polepieces was chosen to give a practical and compact lens design capable of forming an approximately $8 \times$ magnified image with an object-to-image distance of $130 \mathrm{~mm}$. The equipotential distribution of the electrostatic field and the flux density distribution are also shown in Fig. 2. The magnetic lens is designed to produce a negligible magnetic field at the substrate in order to minimize aberrations in the diffraction plane [7]. The lower electric field and the vanishing magnetic field at the sample increases somewhat the focal length of the objective lens and thereby its axial aberrations. The larger aberrations of this lens, however, can still be compensated by a properly designed MAC.

The specialized software package MIRROR DA [15] developed by MEBS, Ltd. has been used for the aberration analysis of the objective lens and of the electron mirrors that are used for aberration correction. The differential algebra-based (DA) software package computes aberrations of electron mirrors of any order and with any symmetry and handles combinations of electron mirrors and electron lenses in a unified way. Results computed with MIRROR DA were cross-checked with those extracted by direct ray tracing [15], a trusted but much slower process, and were shown to be in good agreement. Table 1 shows the computed values of the key objective lens aberration coefficients (referred to the image plane) obtained by MIRROR DA for an electron energy of 10 and $100 \mathrm{eV}$, for an image plane at a distance of $130 \mathrm{~mm}$ from the substrate surface.

\subsubsection{Simulated resolution prediction without aberration correction-}

Simulations of electron-optical properties of the magnetic objective lens have been completed for aberrations up to $5^{\text {th }}$ order in order to understand the resolution limit once the aberration corrector has been implemented. The result of this analysis for an electron energy of 10, 100 and $1000 \mathrm{eV}$ (covering the range of energies used in LEEM, Auger and XPEEM imaging modes) with an initial energy spread of $0.25 \mathrm{eV}$ and a field of view of $2 \mu \mathrm{m}$ on the specimen is shown in Figs. 3a-c. These figures show plots of all aberrations up to $5^{\text {th }}$ order that are larger than $0.1 \mathrm{~nm}$ as a function of the emission angle. The $3^{\text {rd }}$ order geometric and ( $2^{\text {nd }}$ rank) chromatic aberrations are drawn in dashed lines, while $5^{\text {th }}$ order aberrations are drawn in dotted lines. The total blur is then obtained by adding all aberration terms using Gaussian quadrature. Without aberration correction, the blur is limited by diffraction and spherical and chromatic aberrations to approximately $5.5 \mathrm{~nm}$ at $10 \mathrm{eV}, 4.7 \mathrm{~nm}$ at $100 \mathrm{eV}$, and $3.9 \mathrm{~nm}$ at $1000 \mathrm{eV}$ electron energy. At $10 \mathrm{eV}$, the contribution of the spherical and chromatic aberrations are approximately equal, while at $100 \mathrm{eV}$ and higher energies the spherical aberration dominates. 
2.3.2 Simulated resolution prediction with a tetrode MAC-In our previous work $[8,16]$, the tetrode MAC [14] refined by Wan [18] and Tromp [13] was considered for aberration correction in the MAD-LEEM. In a tetrode MAC, the potential of three electrodes can be varied to independently set the focus and simultaneously adjust the primary spherical $\left(\mathrm{C}_{\mathrm{s}}\right)$ and chromatic $\left(\mathrm{C}_{\mathrm{c}}\right)$ aberration coefficients. In addition, the $3^{\text {rd }}$ order coma contribution can be compensated by a field lens placed at the object/image plane of the tetrode MAC. With the tetrode MAC switched on and tuned to compensate the primary spherical and chromatic aberration of the objective lens, the blur is then reduced by more than $2 \times$ to a value of $2.0 \mathrm{~nm}$ at $10 \mathrm{eV}$ electron energy as shown in Fig. 4a. Here, the resolution is limited by diffraction and the nearly balanced 4 th rank chromatic and $5^{\text {th }}$ order spherical aberrations. For higher electron energies, the blur is then limited by diffraction and $5^{\text {th }}$ order spherical aberration to $1.5 \mathrm{~nm}$ at $100 \mathrm{eV}$, a $3 \times$ reduction as shown in Fig. $4 \mathrm{~b}$, and to $0.7 \mathrm{~nm}$ at $1000 \mathrm{eV}$, a $5 \times$ reduction as shown in Fig. 4c. To further improve the resolution at landing energies in the range of a few $\mathrm{eV}$ to $1000 \mathrm{eV}$, it is necessary to reduce the energy spread as well as to correct the now dominant $5^{\text {th }}$ order spherical aberration. It was suggested [8] that the latter could be achieved by replacing the tetrode MAC with a pentode MAC.

2.3.3 Simulated resolution predictions for a pentode MAC-The pentode mirror is a novel aberration corrector that aims to correct not only the primary spherical and chromatic aberration of the objective lens, but also its fifth order spherical aberration, all in one electron-optical element. A layout of the novel pentode MAC is shown in Fig. 5. The pentode MAC consists of five electrodes: a mirror electrode maintained at a potential more negative than the electron source, three intermediate aperture electrodes, and a ground electrode. The potential of the mirror as well as of the three intermediate electrodes can be varied to independently set the focus and simultaneously adjust the $3^{\text {rd }}$ and $5^{\text {th }}$ order spherical $\left(\mathrm{C}_{\mathrm{s} 3}\right.$ and $\left.\mathrm{C}_{\mathrm{s} 5}\right)$ and $2^{\text {nd }}$ rank chromatic $\left(\mathrm{C}_{\mathrm{c}}\right)$ aberration coefficients of the pentode MAC. The pentode MAC focuses the specimen image onto itself, without forming an intermediate image in the MAC. Once the focus is set, the $3^{\text {rd }}$ and $5^{\text {th }}$ order spherical and $2^{\text {nd }}$ rank chromatic aberration coefficients of the pentode MAC are fine-tuned iteratively by adjusting the remaining electrode potentials to cancel the objective lens aberrations. As the aberration coefficients of the objective lens are strongly dependent on the electron energy, the MAC electrode potentials must be tuned for every operating landing energy of interest. Figure 6 displays the pentode MAC axial equipotential distribution needed to compensate the $3^{\text {rd }}$ and $5^{\text {th }}$ order spherical and $2^{\text {nd }}$ rank chromatic aberrations of the objective lens at 10 $\mathrm{eV}$ and $100 \mathrm{eV}$ electron energy. A field lens placed at the object/image plane of the pentode MAC controls the field ray slope and can be utilized to cancel the objective lens $3^{\text {rd }}$ order coma. The blur is now limited by diffraction, $4^{\text {th }}$ rank chromatic aberrations, and $5^{\text {th }}$ order coma. Table 1 shows the computed values of the key pentode MAC aberration coefficients (referred to the image plane) obtained by MIRROR DA for an electron energy of 10 and 100 $\mathrm{eV}$. With a relatively coarse iteration step of $10 \mathrm{~V}$ on the pentode electrodes in the simulation, the leading $3^{\text {rd }}$ order spherical aberration, the $2^{\text {nd }}$ rank chromatic aberration, and the $5^{\text {th }}$ order spherical aberration are all compensated to a fraction of $1 \%$.

At lower electron energies (up to $100 \mathrm{eV}$ ), the blur is limited simultaneously by the 5 th order spherical aberration and 4th rank chromatic aberrations. In order to reduce the blur 
significantly at these energies, it is necessary to simultaneously reduce the energy spread in addition to the correction of the 5th order spherical aberration. At higher electron energies, $(1000 \mathrm{eV})$, the blur is dominated by the 5 th order spherical aberration. Here, it is possible to reduce the blur significantly even without reducing the energy spread. In LEEM mode, where the illumination arm of the microscope is active, the energy spread is reduced by the monochromator (section 2.1.1). In the AES and XPS imaging modes, the energy spread can be reduced by the imaging energy filter (section 2.2.1).

Simulations predict that the pentode MAC reduces the blur by approximately $2 \times$ when compared to the tetrode MAC; that is, the blur drops from $2.0 \mathrm{~nm}$ to $1.1 \mathrm{~nm}$ at $10 \mathrm{eV}$ with an energy spread of $25 \mathrm{meV}$, and from $1.5 \mathrm{~nm}$ to $0.8 \mathrm{~nm}$ at $100 \mathrm{eV}$ electron energy with an energy spread of $100 \mathrm{meV}$, as shown in Fig. 7. At $10 \mathrm{eV}$ electron energy, the energy spread needs to be reduced to $25 \mathrm{meV}$ to achieve a significant improvement, while at $100 \mathrm{eV}$ a larger energy spread of $100 \mathrm{meV}$ is sufficient to significantly reduce the blur. At $1000 \mathrm{eV}$, a significant improvement in reduction can be achieved without reducing the energy spread: the blur drops from $0.7 \mathrm{~nm}$ to $0.5 \mathrm{~nm}$ with an energy spread of $250 \mathrm{meV}$. Even with a larger energy spread of $1 \mathrm{eV}$, the blur can be maintained at $0.7 \mathrm{~nm}$. Hence, at the higher electron energies, the pentode MAC can be used to either improve the resolution in LEEM mode or to significantly increase the selected energy window and thereby the available signal in the energy filtered XPS and AES modes while maintaining sub-nanometer blur.

In the calculations above, the aberration contributions from the transfer optics between the cathode objective lens and the MAC have been neglected. For a detailed column design, all the intermediate optical elements will be taken into account, and the MAC will be tuned to correct the net aberrations of all the elements from the objective lens to the MAC.

\section{DNA sequencing by imaging}

The determination of the entire sequence of DNA bases in a genome is one of the fundamental problems of biology and genome research. Despite the tremendous progress in sequencing technology over the last decade, genome sequencing is still costly and time consuming. One key drawback of current state-of-the-art sequencing technologies is that they identify only 10-1000 bases out of the 3 billion base pairs in the human genome in a given sequence segment or read. The complex repetitive nature of DNA makes the accurate reassembly of the sequence of a full genome from short reads difficult. Another key drawback of these technologies is their relatively large raw read error rate, which necessitates oversampling for important applications like de novo sequencing and resequencing.

In imaging the sequence, the read length is in principle determined by the length of the longest contiguous DNA strand that can be stretched on a flat surface. Transmission Electron Microscopy (TEM) is a high resolution imaging technique that has been proposed for increasing the read length and accuracy of DNA sequences $[18,19]$. In TEM, subnanometer resolution is obtained by impacting the specimen with high energy electrons (80 $-300 \mathrm{keV}$ ), and the images are formed by the electrons transmitted through the specimen. As the individual bases are composed of only light elements ( $\mathrm{H}, \mathrm{C}, \mathrm{O}, \mathrm{N}$ and $\mathrm{P})$, the high 
impact energy necessitates the use of heavy atom labels to provide contrast. The high energy electrons also inflict radiation damage that not only alters the DNA molecules, but can also mobilize the heavy atom labels. The complications associated with reliably labeling the bases and locating the labels along the strand leads to significant read errors. An approach capable of imaging DNA without radiation damage and possibly without labeling is thus highly desirable. The MAD-LEEM approach has several key advantages when compared to TEM techniques: low electron landing energies; precise charge balance; and the potential for label-free nucleotide specific contrast.

The low landing energy of electrons in a LEEM is critical for avoiding radiation damage and achieving high exposure doses, as high energy (>1 keV) electrons cause irreversible damage to biological molecules. Experimental results suggest that DNA is a hardy substance that withstands electron radiation with electron energies in the range from 60 to $230 \mathrm{eV}$, despite a vast dose of $10^{8}$ electrons $/ \mathrm{nm}^{2}$ accumulated over more than one hour [20]. A high electron dose is critical for achieving high throughput, as throughput scales directly with electron dose.

The conductivity of DNA is claimed to vary from that of an insulator to that of a superconductor [21,22], depending on the substrate and the way DNA is attached to the electrodes. When a partially or fully insulating macromolecule is imaged with an electron beam, the imbalance between the arriving and leaving electron flux may cause the DNA strand to charge, resulting in added blur. The charge balance beam incorporated into the MAD-LEEM design prevents the potential charging of individual DNA molecules.

The key remaining feature required for label-free imaging of DNA is the presence of basespecific contrast at low electron energies. DNA consists of two long polymer strands made of sugars and phosphate groups, entwined like vines in the shape of a double helix [23]. Attached to each sugar is one of the four bases: adenine (A), cytosine (C), guanine (G) and thymine (T). Cytosine and thymine are pyrimidines, a class of aromatic six-membered heterocyclic organic compounds with two nitrogen atoms in the ring. Adenine and guanine are double-ringed molecules called purines, consisting of a pyrimidine ring fused to an imidazole ring, a 5-membered ring molecule with two nitrogen atoms in the ring. There are no nitrogen atoms in the sugar-phosphate backbone. In total, the bases $\mathrm{C}$ and $\mathrm{T}$ contain 3 and 2 nitrogen atoms, respectively, and thus can be unambiguously identified by their nitrogen content. A and $\mathrm{G}$ bases both have 5 nitrogen atoms, and thus cannot be distinguished by total nitrogen content alone. However, A and G differ in the nitrogen bonding states as well as in the number of oxygen atoms: A contains 5 oxygen atoms, while G contains 6 . This means that the nitrogen-to-oxygen ratios and nitrogen bonding states can be used in principle to distinguish the individual bases.

\section{Experiments}

The goal of the experimental work is to establish that contrast mechanisms exist at low electron landing energies, from near $0 \mathrm{eV}$ up to a few hundred $\mathrm{eV}$, strong enough to distinguish individual DNA bases or base-pairs. Base-specific contrast is the key ingredient needed to establish the feasibility of the proposed sequencing approach. If base-specific 
contrast does not exist or is weak, the sequencing of unlabeled DNA by the LEEM will become unfeasible, even if the prototype has the desired resolution. It is critical to evaluate the key risks prior to committing time and resources to building a prototype and demonstrating its performance.

Currently, no LEEM can achieve sub-nanometer resolution. In order to gain insight into the contrast that is achievable prior to building the MAD-LEEM instrument, the experimental work has been focused on obtaining contrast values from bulk specimens with synthetic single base (A,C, G or T) DNA strands. The imaging of these oligomers places a lower demand on the required resolution. We performed XPS and AES measurements with commercial systems (PHI Versaprobe XPS and Perkin-Elmer PHI 10-155 AES), which average over a specimen area of typically a few hundred micrometers. The measured reflectivity or emissivity from these bulk samples provides the nominal contrast values. It is believed that these nominal values will describe the inherent contrast of the bases embedded in a sequence and that the effective contrast will be determined by a convolution of the nominal values with the contrast transfer function of the microscope.

\subsection{Specimen preparation}

Gold surfaces were produced by sputtering gold onto silicon wafers using Chromium as an intermediate adhesive layer (Stanford Nanaofabrication Facility). Gold substrates were cleaned in UV-Ozone (Jelight UVO-cleaner 42) for $15 \mathrm{~min}$ followed by immersion in a 1 $\mathrm{mM}$ aminoundecanethiol (Sigma-Aldrich) solution in ethanol for overnight incubation. Subsequently substrates were washed extensively with ethanol and dried in a stream of Argon. Single-stranded homopolymeric oligonuclotides (20mers) were supplied by Integrated DNA Technologies and diluted in deionized water. DNA samples were prepared by spotting $20 \mathrm{uL}$ of a $100 \mathrm{uM}$ oligo solution directly onto the aminoundecanethiol-coated gold substrate and left to dry overnight in a sealed $\mathrm{NaCl}$-saturated Teflon chamber.

\subsection{Spectroscopy}

In addition to electron reflectivity measurements [16], a main thrust of the early phase of this work, X-ray Photoelectron Spectroscopy (XPS) and Auger electron spectroscopy (AES) have also been pursued with encouraging results to distinguish specimens with oligomers containing only one of the four bases. XPS and AES are closely related quantitative, spectroscopic techniques that measure the elemental composition and the chemical and electronic states of a specimen. The spectra are obtained by irradiating the material with a beam of X-rays for XPS and with a beam of electrons for AES, while simultaneously measuring the kinetic energy and number of electrons that escape the specimen. In both methods, low energy electrons are measured, giving rise to comparable depth and sensitivity values, which are respectively on the order of nanometers and of about $0.1 \%$ atomic concentration.

XPS and AES have been used to investigate DNA before to quantitatively characterize the immobilization of thiolated (dT)25 single-stranded DNA on gold [24], to study the structures of thymine and cytosine adsorbed on a $\mathrm{Cu}(110)$ surface [25], and to investigate the quantity of DNA adsorbed on mica surfaces [26]. One of the main obstacles is the lateral 
resolution of the two methods, which is typically in the 10 to $100 \mathrm{~nm}$ range. The proposition is to integrate these techniques into the MAD-LEEM, which will have the requisite resolution, and utilize their elemental and chemical specificity to distinguish the individual nucleotides to achieve sequencing by direct imaging.

4.2.1 XPS and AES results-XPS measurements were performed on a commercial XPS System (PHI Versaprobe) equipped with a monochromatic Al Ka source with a spot size of $100 \mathrm{um}$ and a hemispherical electron energy analyzer. The reported peak counts and binding energies are based on the analyzer count and energy calibration. AES measurements were performed on a commercial AES system (Perkin-Elmer PHI 10-155) operating at $3 \mathrm{keV}$ and with an emission current of $1 \mathrm{~mA}$. The AES system is equipped with a cylindrical mirror electron energy analyzer, with an energy resolution of $0.5 \mathrm{eV}$. The Auger spectra were numerically differentiated to remove the large background of secondary electrons.

Figure 8 shows XPS (a) and differentiated Auger (b) N 1s spectra for a set of four samples prepared by immobilizing 20 mers of A, C, G, and T on a Au-coated Si substrate functionalized with a monolayer of aminoundecanethiol. In order to account for the varying DNA coverage of the individual samples, the XPS spectra were normalized to the phosphor signal, which is present in equal amounts for all four nucleotides. In AES the phosphor peaks were rather noisy. Consequently, the alignment was performed with respect to the oxygen peak. The nitrogen content in the XPS spectra is derived from the area under the nitrogen peak, whereas in the AES spectra, the nitrogen content is derived from the difference between the maximum and minimum of the derivative spectrum around the expected peak position. Both the XPS and AES spectra clearly distinguish the purine bases (A and $\mathrm{G}$ ) from the pyrimidine bases ( $\mathrm{C}$ and $\mathrm{T}$ ): the larger nitrogen content in the purines (5 nitrogen atoms per base) results in stronger nitrogen signals when compared to the pyrimidines ( $\mathrm{C}$ and $\mathrm{T}$, which have 3 and 2 nitrogen atoms per base, respectively). Detailed, quantitative analysis of the XPS and AES spectra [27] shows that the impact of the elemental composition and bonding states on the spectra are in good qualitative agreement with theory. The nitrogen-to-oxygen ratios derived from XPS and AES spectra thus offer sufficient contrast to distinguish the bases; however, the need to acquire images at two characteristic energies will increase the time for the data acquisition, which will reduce the throughput.

Surprisingly, further detailed analysis of the XPS spectra shows that the nitrogen spectrum itself may be sufficient to unambiguously distinguish the individual bases. This realization can be understood by focusing on the small energy window from 398 to $400 \mathrm{eV}$ marked by a dashed box in Fig. 8a. Within this energy window, the nitrogen signals are increasing in nearly equal steps, starting with the minimum nitrogen content for $\mathrm{T}$, followed by increased content for $\mathrm{C}$ and $\mathrm{G}$, and reaching a maximum for $\mathrm{A}$. We attribute this linear dependence for nitrogen to the presence of the double nitrogen-carbon bonds present in differing amounts in the individual bases: $\mathrm{T}$ has no double nitrogen-carbon bonds, while $\mathrm{C}$ has one, $\mathrm{G}$ has two and A has three. Consequently, in the XPS imaging mode, a single energy-filtered image acquired at the lower binding energy shoulder of the nitrogen peak is sufficient for distinguishing the individual bases, which significantly simplifies the image processing and increases the throughput. 


\section{Modeling the image contrast of nucleotides in a DNA sequence}

Here, a model is presented for converting the nominal electron intensity measurements, those made on bulk DNA, into the expected contrast of the individual bases in a sequence. Given that the existing spectroscopic measurements are made on the nucleotide unit as a whole, the nucleobase is lumped together with its associated sugar and phosphate groups in the backbone. Each strand is modeled as having discrete nucleotide units spaced along the helix axis by the rise per base pair, $\gamma$. The effects of the optical blur and pixelation on the nucleotide contrast are modeled by assuming that the units are two-dimensional intensity objects, emitting the electrons uniformly over a rectangular cross-section. Furthermore, the optical blur in mapping these objects onto the detector is modeled by a two dimensional Gaussian point-spread function (PSF). The mathematical details of this model have been provided elsewhere [8]. In short, the rectangular cross-section combined with the Gaussian PSF separates the two dimensional image profile into the product of intensities, $I_{x} I_{y}$, where $x$ describes the direction along the helix axis, $y$ describes the direction across this axis in the plane of the substrate, and $I_{i}=I\left(n_{i}, p_{i}, \varepsilon_{i}, l_{i}\right)$ with

$$
I(n, p, \varepsilon, l) \equiv \frac{1}{2 p} \sum_{s_{l}, s_{p} \in \pm 1} s_{l} s_{p}\left[u \operatorname{erf} u+\frac{1}{\sqrt{\pi}} e^{-u^{2}}\right]_{u=n p-s+\frac{1}{2}\left(s_{l} l+s_{p} p\right)}
$$

Here, $n$ represents the pixel number, $p$, the pixel size, $\varepsilon$, the pixel offset relative to the feature center, and $l$, the feature length, with all quantities normalized to $\sqrt{2} \sigma$, where $\sigma$ is the standard deviation in the Gaussian PSF. It is assumed that the pixel array on the detector is aligned with respect to the rectangular feature.

\subsection{Double-stranded DNA}

The typical, aqueous form of double-stranded DNA, B-DNA, comprises two intertwined helices with a diameter of $2.0 \mathrm{~nm}$. The nucleotides are spaced along the helix with a rise per base pair of $\gamma=0.34 \mathrm{~nm}$. The helical pitch of B-DNA is $h=3.4 \mathrm{~nm}$, thereby containing 10 pairs per helical turn. The base pair tilt angle of $1.2^{\circ}$ is neglected here, making the axis running through the pair normal to the helix axis. Furthermore, each nucleotide is modeled to have a circular cross-section with a diameter $l_{y}$ as viewed from the helix axis. The nucleotide is imparted a height, $l_{x}$, along the axis, which is set to a fraction of $\gamma$. As such, each nucleotide presents a rectangular cross-section to the beam with a constant area, $l_{x} \times l_{y}$, regardless of its orientation.

5.1.1 The helical geometry-A helix with pitch $h$ and radius $a$ is described in complex notation by

$$
\boldsymbol{R}(x, \phi ; h, a)=y+i z=a e^{i\left(2 \pi \frac{x}{h}+\phi\right)}
$$

with the $z$ coordinate representing the direction of the electron beam (collinear with the surface-normal of the substrate). The phase $\varphi$ defines the vector $\boldsymbol{R}$ in the reference, $x=0$, plane. If the two intertwined helices form a symmetric double helix, then the phase 
difference between them is $\Delta \varphi=\pi$. In general, however, the major and minor grooves of the double helix are not equal, and the second strand is found to be rotated by the angle

$$
\Delta \phi=\frac{2 \pi}{1+\rho}
$$

where $\rho$ is the ratio of the major to the minor groove. For B-DNA, $\rho \approx 2$, making $\Delta \varphi \approx$ $2 \pi / 3$.

Given that the $n$ 'th pair is located in the plane $x_{n}=n \gamma$, the lateral positions where the nucleotides meet the strands are found as

$$
\boldsymbol{R}_{n}^{ \pm}=\boldsymbol{R}\left(n \gamma, \phi \pm \frac{\Delta \phi}{2} ; h, a\right)
$$

where the \pm superscripts denote the two strands. The centers of the two nucleotides, $\boldsymbol{r}_{n}^{ \pm}$, are assumed to be located at $1 / 4$ and $3 / 4$ along the cord connecting the strands; that is,

$$
\boldsymbol{r}_{n}^{ \pm}=\boldsymbol{R}_{n}^{-}+\frac{1}{2}\left(1 \pm \frac{1}{2}\right)\left(\boldsymbol{R}_{n}^{+}-\boldsymbol{R}_{n}^{-}\right)=\left(1+\frac{1}{2}\left(1 \pm \frac{1}{2}\right)\left(e^{i \Delta \phi}-1\right)\right) \cdot \boldsymbol{R}\left(n \gamma, \phi-\frac{\Delta \phi}{2} ; h, a\right)
$$

For the general non-symmetric case, where $\Delta \varphi \neq \pi$, the chord will not pass through the center of the cylinder encapsulating the double helix. Last, the diameter of the nucleotide is set equal to half the cord length so that the pair just touch, making

$$
l_{y}=\frac{1}{2}\left|\boldsymbol{R}_{n}^{+}-\boldsymbol{R}_{n}^{-}\right|=\frac{a}{2}\left|e^{i \Delta \phi}-1\right|=a \mid \sin \frac{\Delta \phi}{2}
$$

The model ds-DNA geometry is shown in Fig. 9a using the parameters characterizing BDNA. Here, the height of the nucleotide units is set equal to half of the rise per base pair; that is, $l_{x}=\gamma / 2$.

\subsection{The nominal intensity}

The nucleobase pair comprises one purine, adenine or guanine, paired with a pyrimidine, thymine or cytosine, respectively. In the XPS measurements, the nominal emissivities of the four bases were found to increase in the order $T, C, G, A$. Hence, the nominal emissivity of the purine in the base-pair is labeled as $\eta_{i}^{+}$, and that of the pyrimidine is labeled as $\eta_{i}^{-}$, where $i=A T$ describes the pair Adenine- Thymine and $i=G C$ describes the pair GuanineCytosine. In the regions outside the span of the nucleotides, the intensity is set to that of the background substrate, $\eta_{0}$.

To simplify the image analysis, an archetype imaging condition is assumed, in which the average nominal intensities for the two base pairs are set equal. In addition, the nominal intensity of the substrate, $\eta_{0}$, is also set to this average value; that is, 


$$
\eta_{i}^{ \pm}=\eta_{0}\left(1 \pm c_{i}\right)
$$

with $c_{i}$ describing the base pair contrast,

$$
c_{i} \equiv \frac{\eta_{i}^{+}-\eta_{i}^{-}}{\eta_{i}^{+}+\eta_{i}^{-}}
$$

Also, in the region highlighted in Fig. 9b, where the base pairs overlap as viewed by the beam, the nominal intensity is averaged:

$$
\bar{\eta}_{i} \equiv \frac{1}{2}\left(\eta_{i}^{+}+\eta_{i}^{-}\right)=\eta_{0}
$$

Consequently, the region of overlap in this model will have the nominal intensity of the substrate. This region is determined by the orientation of the pair relative to the electron beam. Defining $\Delta \theta \equiv 2 \pi \gamma / h$ as the rotation angle of the helix per base pair, the orientation of the $n$ 'th pair is found as

$$
\theta_{n}=n \Delta \theta+\theta_{0}
$$

where $\theta_{0}$ is the orientation of the initial pair. This angle is defined to be 0 when the chord connecting the base pair is perpendicular to the beam direction. The extent of overlap for the $n$ 'th pair is then given by

$$
\bar{l}_{n}=2 l_{y} \sin ^{2}\left(\frac{\theta_{n}}{2}\right)
$$

which maximizes to the value of the nucleotide diameter, $l_{y}$, when the chord and the beam are collinear $\left(\theta_{n}=\pi / 2\right)$.

\subsection{Simulated XPS images}

The effects of optical blur, pixelation, and noise on the image of the nucleotides in a sequence of B-DNA are simulated for a likely XPS condition, characterized by an electron density at the sample that is equal to

$$
n=100 e^{-} / \AA^{2}
$$

The density determines the number of electrons per pixel, which impacts the shot noise. The pair intensity contrast is estimated from the nitrogen (1s) XPS measurements to be $c_{T A}=0.6$ and $c_{C G}=0.2$. It should be noted that the nitrogen atoms reside solely in the nucleobases, making the phosphate-sugar backbone of DNA transparent in this measurement. 
The image of B-DNA is compared to its denatured form [28], where the two strands remain separated over a length scale that is comparable to 100 base pairs. The aim is to produce the denatured form of DNA in preparation for imaging via the method of molecular combing [29], for example. In addition to being mostly unwound, the ss-form has a rise per base pair that is a factor of 1.7 greater than the B-form, which reduces the demand on the resolving power of the microscope. Here, however, an equal comparison is made by modeling the ssform with $\gamma=0.34 \mathrm{~nm}$ in order to highlight the effect of the helicity on the nucleotide contrast in the image. The strand separation for ss-DNA is assumed to be that of the B-form: $2 a=2.0 \mathrm{~nm}$. The center position of each base in ss-DNA is allowed to randomly fluctuate about the strand location within the range $\pm 0.5 a= \pm 0.5 \mathrm{~nm}$. The diameter of the nucleotides, $l_{y}$, in the ss-form is set equal to half of the separation or $a=1.0 \mathrm{~nm}$. For both forms, the extent of the nucleotides along the axis, $l_{x}$, is set equal to half of the rise per base pair: $l_{x}=$ $\gamma / 2$.

Figures 10 and 11 show the simulated images of both B-DNA and its ss-form spliced vertically. The two figures compare images taken with $1 \AA$ and $2 \AA$ pixels. Each figure contains images taken with increasing optical blur spliced from left to right. The optical blur, $d$, is defined as the PSF diameter that contains $99 \%$ of the current; that is,

$$
d=\sqrt{-8 \ln (1-0.99)} \cong 6.07 \sigma
$$

where $\sigma$ is the standard deviation of the Gaussian PSF. The pixel noise is derived from adding the shot noise in quadrature to the noise in the overall system (due to the electronics, for example). Here, the noise is assumed to be dominated by shot noise.

In the helical form, the partial screening of the base pair as the axis connecting the pair becomes collinear with the electron beam is evident. Regardless of the form, the observed rapid degradation in the nucleotide contrast with increasing optical blur makes subnanometer resolution key for reducing the sequence error rate. The extended rise per base pair of the actual denatured form of DNA will lessen the effect of the optical blur.

\subsection{Estimating the sequence error rate}

To estimate the way in which the error rate scales with the imaging and feature parameters, a model sequence is chosen that contains two alternating features whose center positions remain aligned. Here, the dynamic range of the gray levels is taken to be infinite.

Consequently, no matter the degree to which the imaging process degrades the contrast between the two features, the intensity of the bright and dark features will remain above and below their mean value, respectively. Therefore, without any pixel noise, the features would be determined without error. However, shot noise introduces gray level fluctuations, which may reduce, for example, the gray level of the bright feature below the average value thereby imparting to this feature the wrong label. For a given number of electrons per pixel, $N$, and gray level intensities for the bright, $I_{+}$, and dark features, $I_{-}$, the probability $F$ that the pixel gray level of either feature registers correctly has been calculated [8] as 


$$
F=\frac{1}{2}(1+\operatorname{erf} \chi)
$$

with

$$
\chi \equiv \sqrt{\frac{N}{2}} \frac{I_{+}-I_{-}}{I_{+}+I_{-}}
$$

and

$$
\frac{I_{+}-I_{-}}{I_{+}+I_{-}}=\rho c
$$

where $c$ is the nominal contrast and $\rho$ describes the loss of contrast from the imaging process. For optical blur values greater than the rise per feature $(d>\gamma)$, the contrast loss factor, $\rho$, for an array of alternating features may be approximated by the dominant Fourier mode associated with the periodicity along the strand to give

$$
\rho \cong 4 \frac{l_{x}}{2 \gamma} \frac{l_{y}}{\sqrt{2 \pi} \sigma^{*}} e^{-\left(\pi \frac{\sqrt{2} \sigma}{2 \gamma}\right)^{2}} \operatorname{sinc}\left(\pi \frac{p}{2 \gamma}\right) \operatorname{sinc}\left(\pi \frac{l_{x}}{2 \gamma}\right)
$$

where

$$
\operatorname{sinc} u \equiv \frac{1}{u} \sin u
$$

$l_{x}$ and $l_{y}$ are the feature length and width, respectively, $p$ is the pixel size, and

$$
\sigma^{*} \equiv \sqrt{1+\frac{p^{2}+l_{y}^{2}}{12 \sigma^{2}}} \sigma
$$

is the augmented standard deviation arising from the convolution (in the direction normal to the array) of the PSF with the feature width and the pixel size. Here, the feature and the pixel centers are assumed to be aligned. The goodness of the approximation for $\rho$ is depicted in Fig. 12a, which graphs it against the numerically derived result for various values of the independent variables. The relative error between the two maximizes to $\sim 50 \%$ as $l_{y} \rightarrow \infty$ owing mostly to the fact that

$$
\frac{l_{y}}{\sqrt{2 \pi} \sigma^{*}} \rightarrow \sqrt{6 / \pi}
$$


which overestimates the correct limit of 1.0. If the upper bound of this factor is pinned to 1.0 , then the maximum relative error drops to $34 \%$ for $d=\gamma$ and to $13 \%$ for $d=1.5 \gamma$.

Noting that $N=n p^{2}$ for square pixels, a dominant, sinusoidal $p$-dependence for $\chi$ is revealed, which is maximized for $p=\gamma$ to give

$$
\chi \cong c \frac{A}{\gamma^{2}} \sqrt{\frac{N}{2}} \cdot g(\sigma / \gamma)
$$

with $A \equiv l_{x} l_{y}$ equal to the feature area and

$$
g(\sigma / \gamma) \equiv \frac{2}{\sqrt{\pi}}\left(1+\frac{\gamma^{2}}{6 \sigma^{2}}\right)^{-\frac{1}{2}} \frac{\sqrt{2} \gamma}{\pi \sigma} e^{-\left(\frac{\pi \sigma}{\sqrt{2} \gamma}\right)^{2}}
$$

Here, $\operatorname{sinc}\left(\pi l_{x} / 2 \gamma\right)$ has been approximated as 1.0 and $l_{y}$ has been set to $\gamma$ in the expression for $\sigma^{*}$.

The function $g$ describes the loss of contrast, which degrades exponentially with the square of the optical blur. For vanishing $\chi$, the error rate, defined as $1-F$, approaches 0.5 as it becomes equally likely for the features to register above or below the mean value. The coefficient multiplying the function $g$ to give $\chi$ is a product of three parameters: the nominal contrast; the fraction of the pixel area $\left(p^{2}=\gamma^{2}\right)$ taken by the feature; and the square-root of the number of electrons per pixel. The triple product is graphed against the error rate for a few ratios of the optical blur to the feature pitch in Fig. 12b. The curve for $d=\gamma$ (corresponding to a marginally valid approximation for $\rho$ ) is essentially the result that is found with no optical blur (shown by circles), where

$$
\chi \rightarrow c \frac{A}{p^{2}} \sqrt{\frac{N}{2}}
$$

Figure 12B indicates that for a feature pitch of $\gamma \sim 5 \AA$ and an optical blur of $d \sim 1 \mathrm{~nm}$, the value of the triple product must fall in the range of $[5.5,8]$ to produce error rates in the range of $\left[10^{-3}, 10^{-6}\right]$, respectively. For the areal density of $100 \mathrm{e} / \AA^{2}$, which gives $N=2500$ electrons per pixel, the above range for the triple product translates to a contrast cross section that is in the range of

$$
c A \sim[3.0,4.0] \AA^{2}
$$

If it is assumed that the feature cross section resides within the area encapsulated by the square of the feature pitch $\left(25 \AA^{2}\right)$, then the minimum required contrast for achieving the aforementioned error rates falls in the range

$$
c \sim[0.12,0.16]
$$




\section{Summary and Conclusion}

Recent advancements in the development of MAD-LEEM, a novel microscopy technique utilizing a monochromator, aberration corrector, energy filter and dual-beam electron illumination, have been presented. The key new features are the pentode mirror aberration corrector, the knife-edge based imaging energy filter, the addition of the photoemission electron microscopy (PEEM) imaging mode, and the modified and improved objective lens. Detailed simulations of the system performance based on these new features have been undertaken showing significant improvements. Simulations of the electron-optical properties of the LEEM objective lens have been completed including aberrations up to $5^{\text {th }}$ order in order to understand the resolution limit with aberration correction. The analysis of electrostatic electron mirrors shows that their negative spherical and chromatic aberration coefficients can be tuned over a large parameter range and can be used to compensate the aberrations of the LEEM objective lens for a range of electron energies. A novel mirror aberration corrector, an electrostatic pentode mirror, combined with the monochromator has been proposed to further improve the resolution by correcting the $5^{\text {th }}$ order spherical aberration. While a tetrode MAC reduces the optical blur to $2.0 \mathrm{~nm}$ at $10 \mathrm{eV}, 1.5 \mathrm{~nm}$ at 100 $\mathrm{eV}$, and $3.9 \mathrm{~nm}$ at $1000 \mathrm{eV}$ electron energy, the pentode $\mathrm{MAC}$ reduces the blur further to 1.1 $\mathrm{nm}$ at $10 \mathrm{eV}, 0.8 \mathrm{~nm}$ at $100 \mathrm{eV}$, and $0.5 \mathrm{~nm}$ at $1000 \mathrm{eV}$ electron energy.

A detailed approach for sequencing DNA by imaging in a LEEM has been proposed. In addition to using reflective electrons, two well-established spectroscopic techniques, X-ray Photoelectron Spectroscopy and Auger electron spectroscopy, have been utilized to distinguish samples with oligomers containing only one of the four bases. Contrast simulations of a model, rectangular nucleotide base geometry utilizing the minimum experimental contrast obtained in XPS predict that images will yield a relatively low error rate of $10^{-6}$ when sequencing ss-DNA if the microscope resolution is improved to give blur values of $1 \mathrm{~nm}$ or less. The requirement on the nominal contrast may be relaxed (or equivalently, the error rate may be lowered) with sub-nm resolution, which according to simulations of the MAD-LEEM electron optics can be achieved by incorporating a pentode MAC and a monochromator. This approach thus has promise to significantly improve the performance of a LEEM for DNA sequencing as well as for a wide range of applications in the biosciences, material sciences, and nanotechnology, where nanometer scale resolution and analytical capabilities are required.

\section{Acknowledgments}

This project was supported by Grant Number R43HG006303 from the National Human Genome Research Institute (NHGRI). The work was partially performed at the National Center for Electron Microscopy, supported by the Office of Science, Office of Basic Energy Sciences, of the U.S. Department of Energy under Contract No. DEAC02-05CH11231. The content is solely the responsibility of the authors and does not necessarily represent the official views of the NHGRI or the National Institutes of Health. The authors would also like to thank T.H.P. Chang for his continuous support and numerous suggestions leading to this paper.

\section{REFERENCES}

1. Krivanek OL, Ursin JP, Bacon NJ, Corbin GJ, Dellby N, Hrncirik P, Murfitt MF, Own CS, Szilagyi ZS. Phil. Trans. R. Soc. A. 2009; 367:1. 
2. Kaiser U, Biskupek J, Meyer JC, Leschner J, Lechner L, Rose H, Stöger-Pollach M, Khlobystov AN, Hartel P, Müller H, Haider M, Eyhusen S, Benner G. Ultramicroscopy. 2011; 111:1239. [PubMed: 21801697]

3. Danilatos GD, Robinson VNE. Scanning. 1979; 2:72.

4. Telieps W, Bauer E. Ultramicroscopy. 1985; 17:57.

5. Hibino H, Kageshima H, Maeda F, Nagase M, Kobayashi Y, Kobayashi Y, Yamaguchi H. eJ.Surf.Sci. Nanotech. 2008; 6:107.

6. Rose HH. Sci. Technol. Adv. Mater. 2008; 9:014107.

7. Mankos, M.; Spasov, V.; Munro, E. Advances in Imaging and Electron Physics. Hawkes, Peter W., editor. Vol. 161. 2010. p. 1

8. Mankos M, Shadman K. Ultramicroscopy. 2013; 30:13. [PubMed: 23582636]

9. Mankos, M. U.S. Patent. No. 8,870,172. 2012 May 22.

10. Mankos, M. U.S. Patent. No. 8,334,508.

11. Altman MS. J. Phys.: Condens. Matter. 2010; 22:084017. [PubMed: 21389393]

12. Schmidt, Th; Marchetto, H.; Le'vesque, PL.; Groh, U.; Maier, F.; Preikszas, D.; Hartel, P.; Spehr, R.; Lilienkamp, G.; Engel, W.; Fink, R.; Bauer, E.; Rose, H.; Umbach, E.; Freund, H-J. Ultramicroscopy. 2010; 110:1358. [PubMed: 20692099]

13. Tromp RM, Hannon JB, Ellis AW, Wan W, Berghaus A, Schaff O. Ultramicroscopy. 2010; 110:852. [PubMed: 20395048]

14. Preikszas D, Rose H. Journal of Electron Microscopy. 1997; 1:1.

15. Munro E, Rouse J, Liu H, Wang L. J. Vac. Sci. Technol. 2008; B 26:2331.

16. Mankos M, Shadman K, N'Diaye A, Schmid A, Persson H, Davis R. J. Vac. Sci. Technol. B. 2012; 30:06F402.

17. Wan W, Feng J, Padmore HA, Robin DS. Nucl. Instr. Meth. Phys. Res. 2004; A519:222.

18. Bell DC, Thomas WK, Murtagh KM, Dionne CA, Graham AC, Anderson JE, Glover WR. Microsc. Microanal. 2012:1.

19. Andregg, W.; Andregg, M. U.S. Patent Application. No. 20100267152. 2010 Oct 21.

20. Germann M, Latychevskaia T, Escher C, Fink H-W. Phys. Rev. Lett. 2010; 104:095501. [PubMed: 20366992]

21. Endres RG, Cox DL, Singh RRP. Rev. Mod. Phys. 2004; 76:195.

22. Guo X, Gorodetsky AA, Hone J, Barton JK, Nuckolls C. Nat. Nanotechnol. 2008; 3:163. [PubMed: 18654489]

23. Watson JD, Crick F. Nature. 1953; 171:737. [PubMed: 13054692]

24. Petrovykh D, Kimura-Suda H, Whitman LJ, Tarlov MJ. J. Am. Chem. Soc. 2003; 125:5219. [PubMed: 12708875]

25. Furukawa M, Fujisawa H, Katano S, Ogasawara H, Kim Y, Komeda T, Nilsson A, Kawai M. Surface Science. 2003; 532-535:261.

26. Rabke CE, Wenzler LA, Beebe TP Jr. Scanning Microscopy. 1994; 8(3):471. [PubMed: 7747152]

27. To be submitted to ACS Nano.

28. Rouzina I, Bloomfield VA. Biophys. J. 2001; 80:882. [PubMed: 11159455]

29. Bensimon D, Simon AJ, Croquette V, Bensimon A. Phys. Rev. Lett. 1995; 74:4754. [PubMed: 10058590] 
- We present a LEEM with a monochromator, aberration corrector, energy filter and two electron beams

- We analyze objective lens aberrations up to 5th order with and without aberration correction

- A pentode mirror corrector combined with a monochromator greatly reduces blur

- We present experimental data from bulk DNA specimens demonstrating that base specific contrast is available with X-ray photoemission, Auger, and reflected electrons.

- Contrast simulations utilizing the minimum experimental contrast predict that images will yield a relatively low error rate are obtainable 


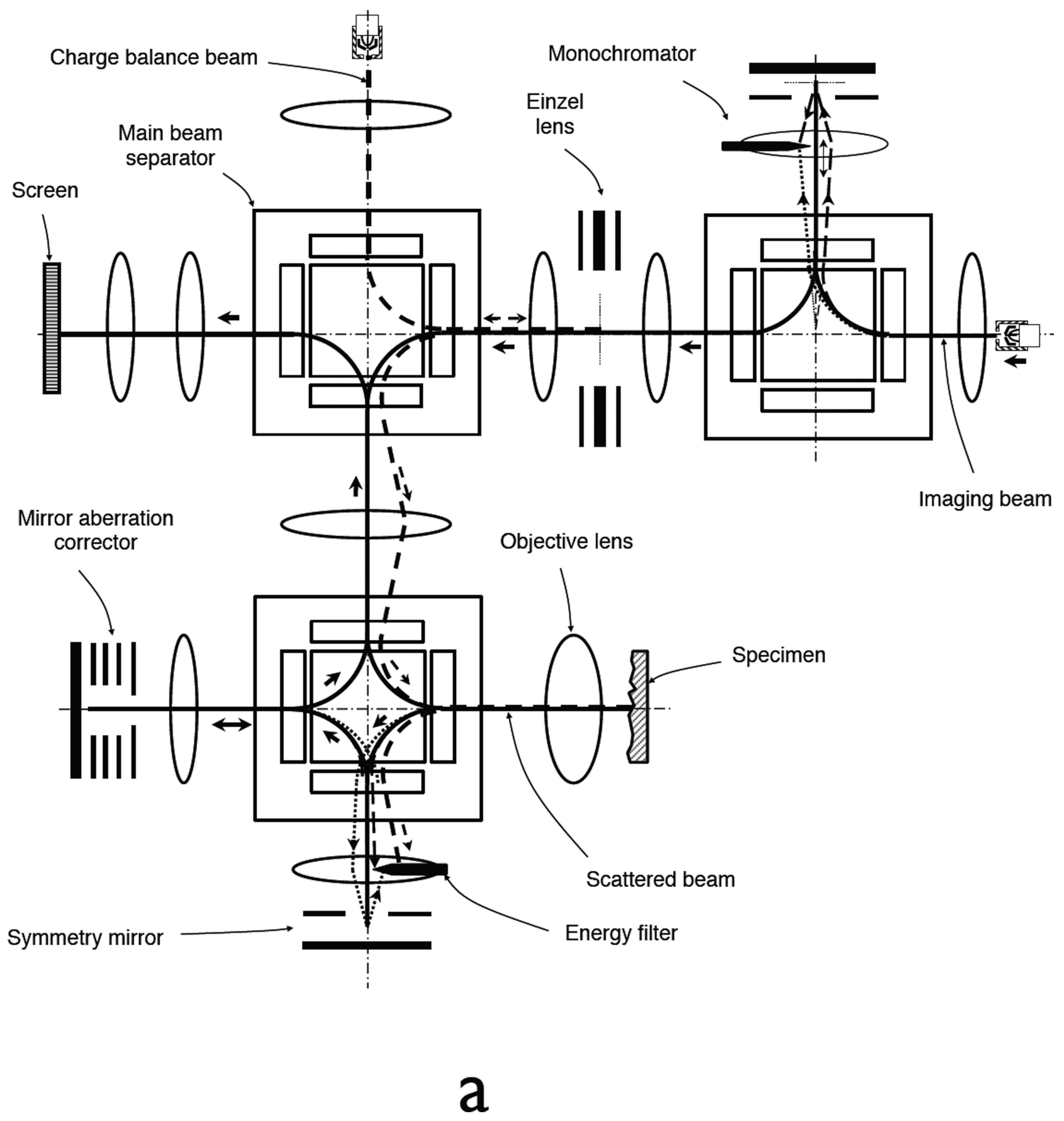




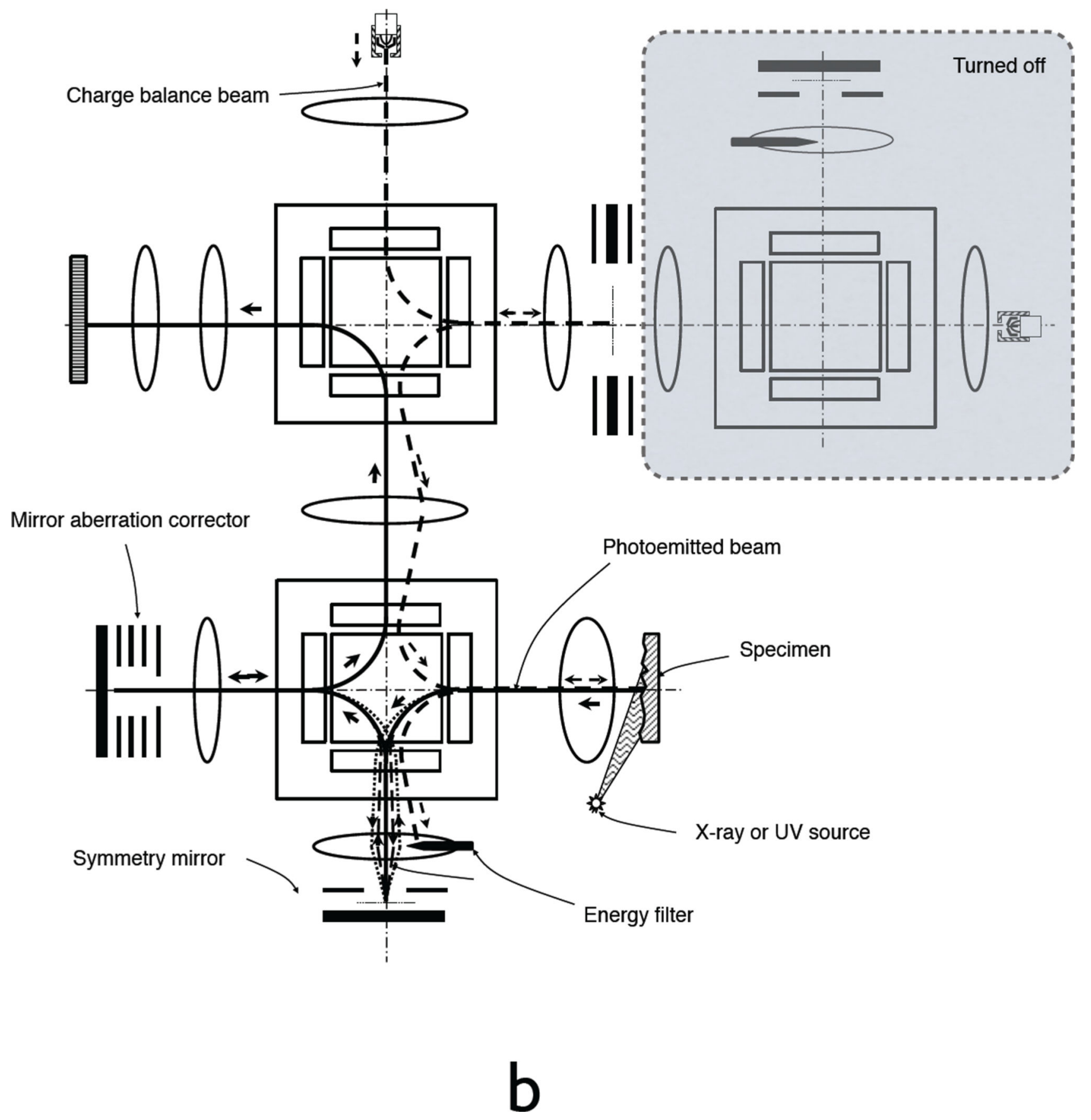

Figure 1.

Schematic layout of a MAD-LEEM column for imaging in dual beam LEEM (a) and (X)PEEM (b) mode. 


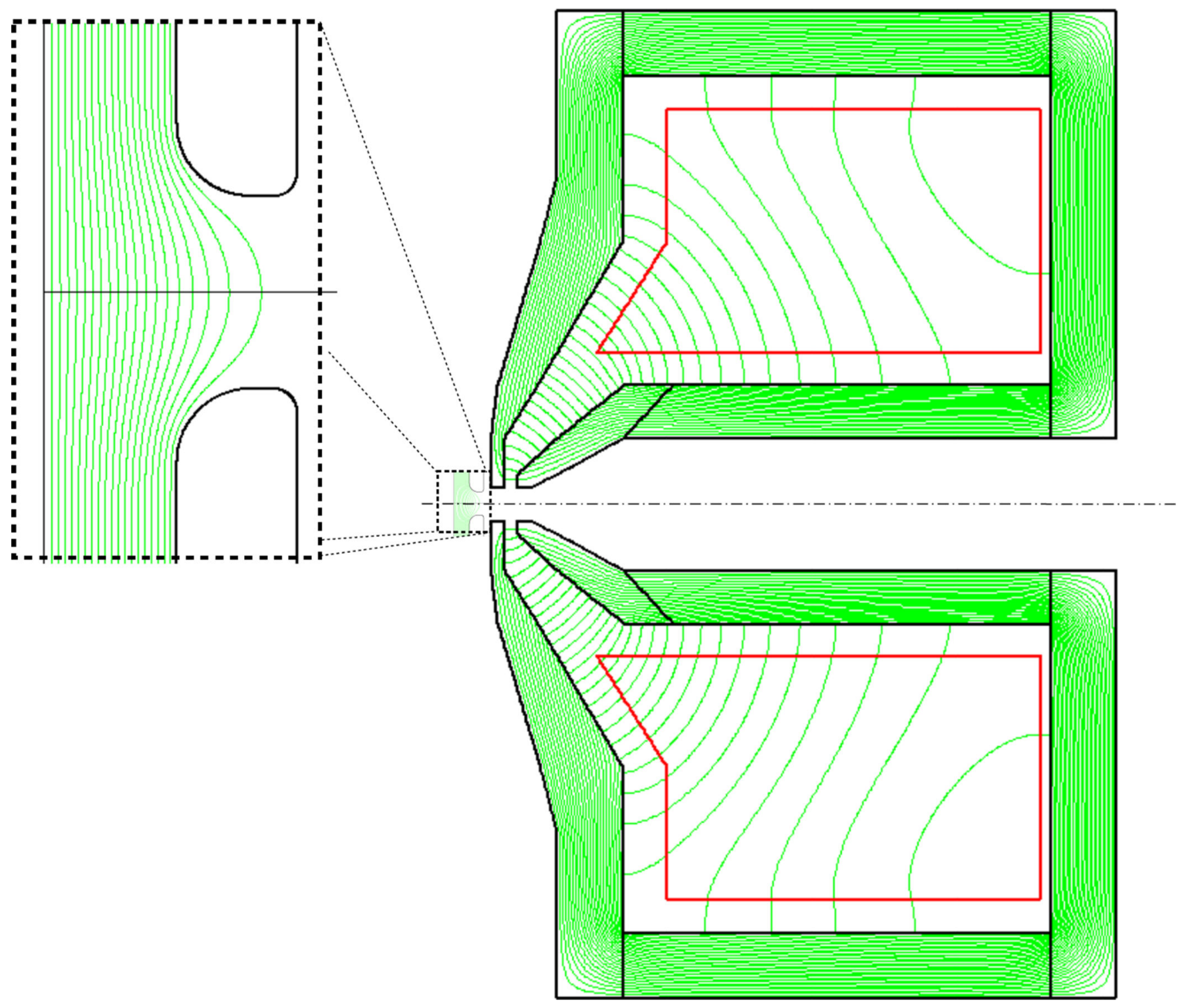

Figure 2.

Geometry, and equipotential and flux density distributions of a magnetic LEEM objective lens, with zoomed-in view of equipotential distrubition of the electrostatic lens. 


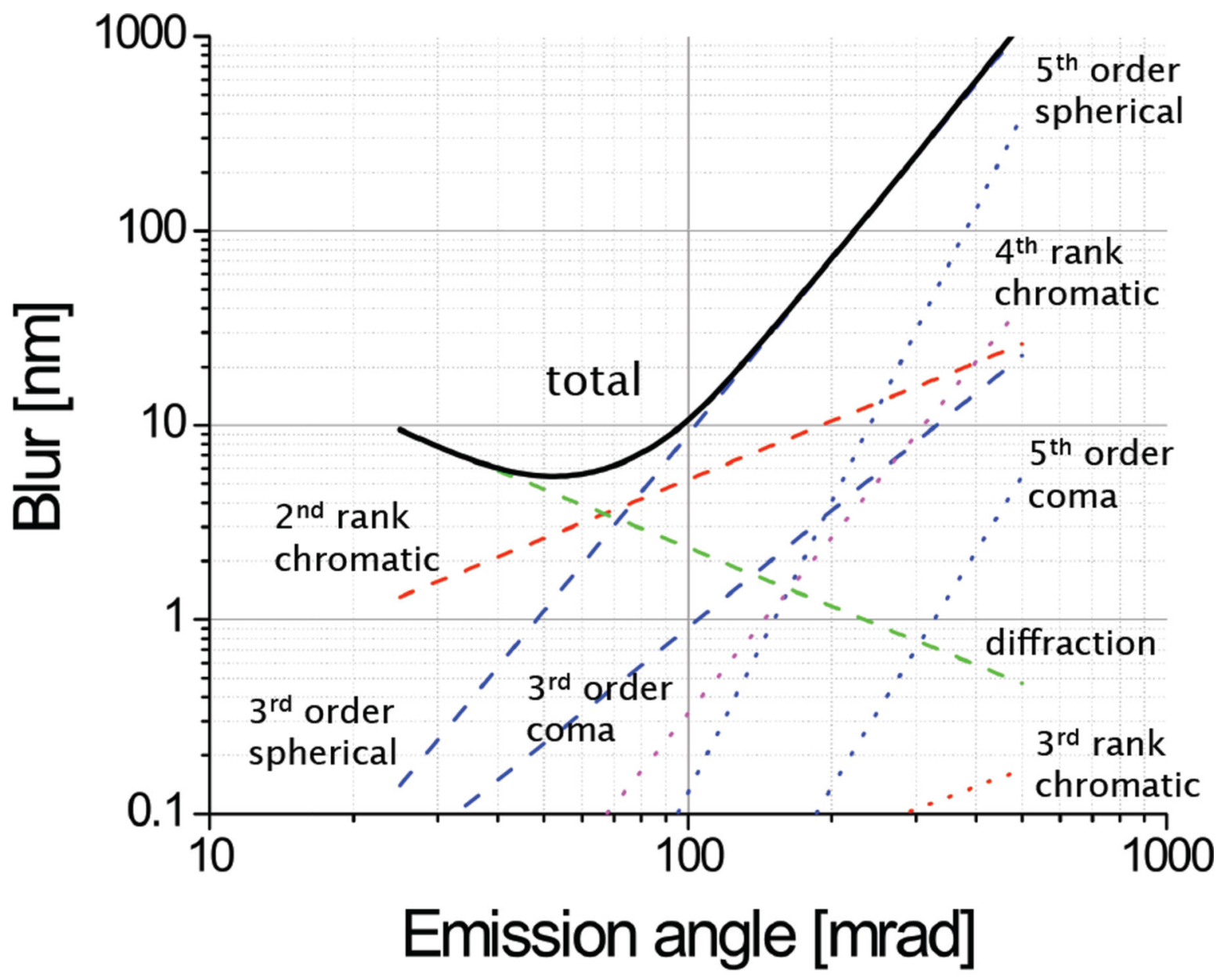

a 


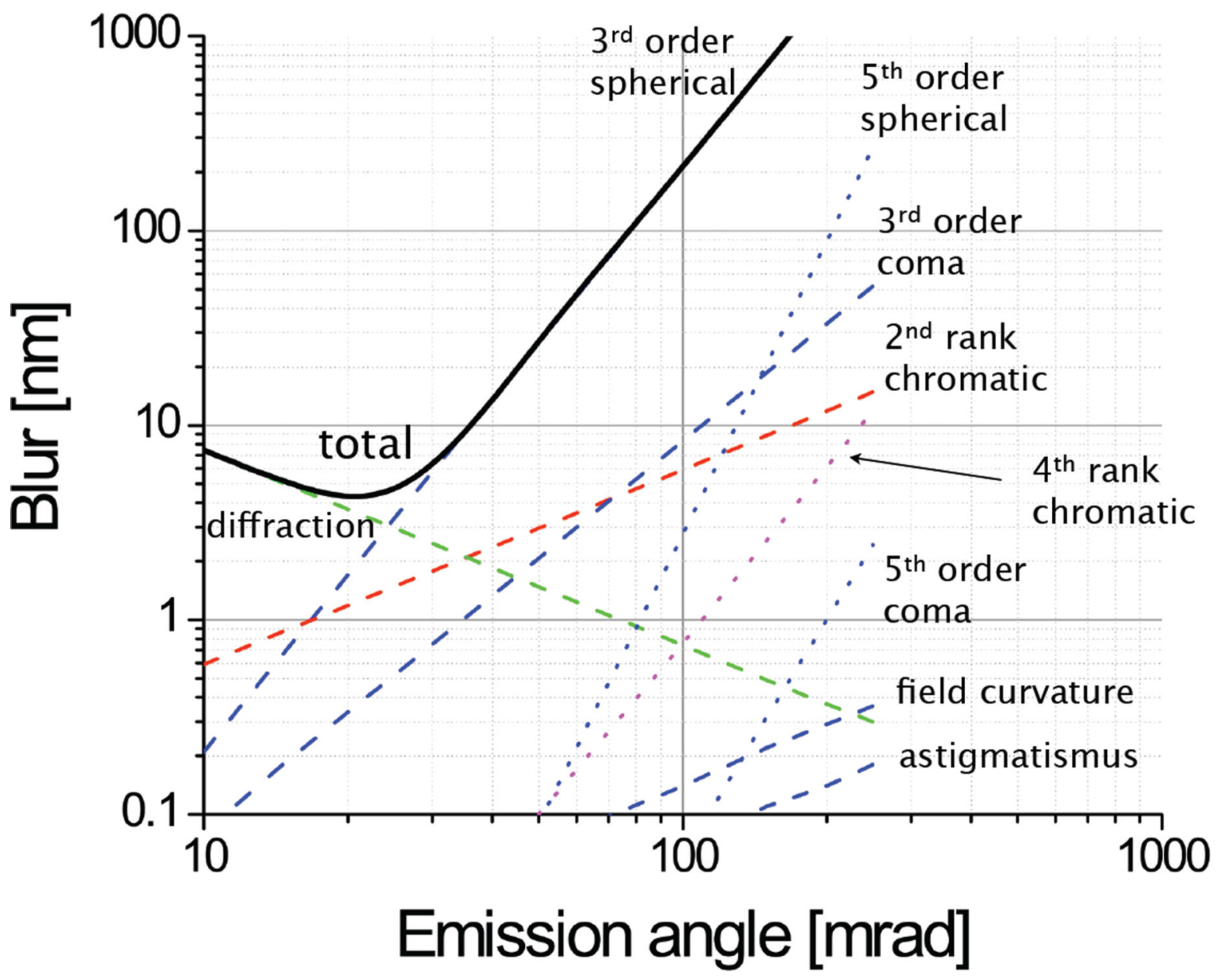



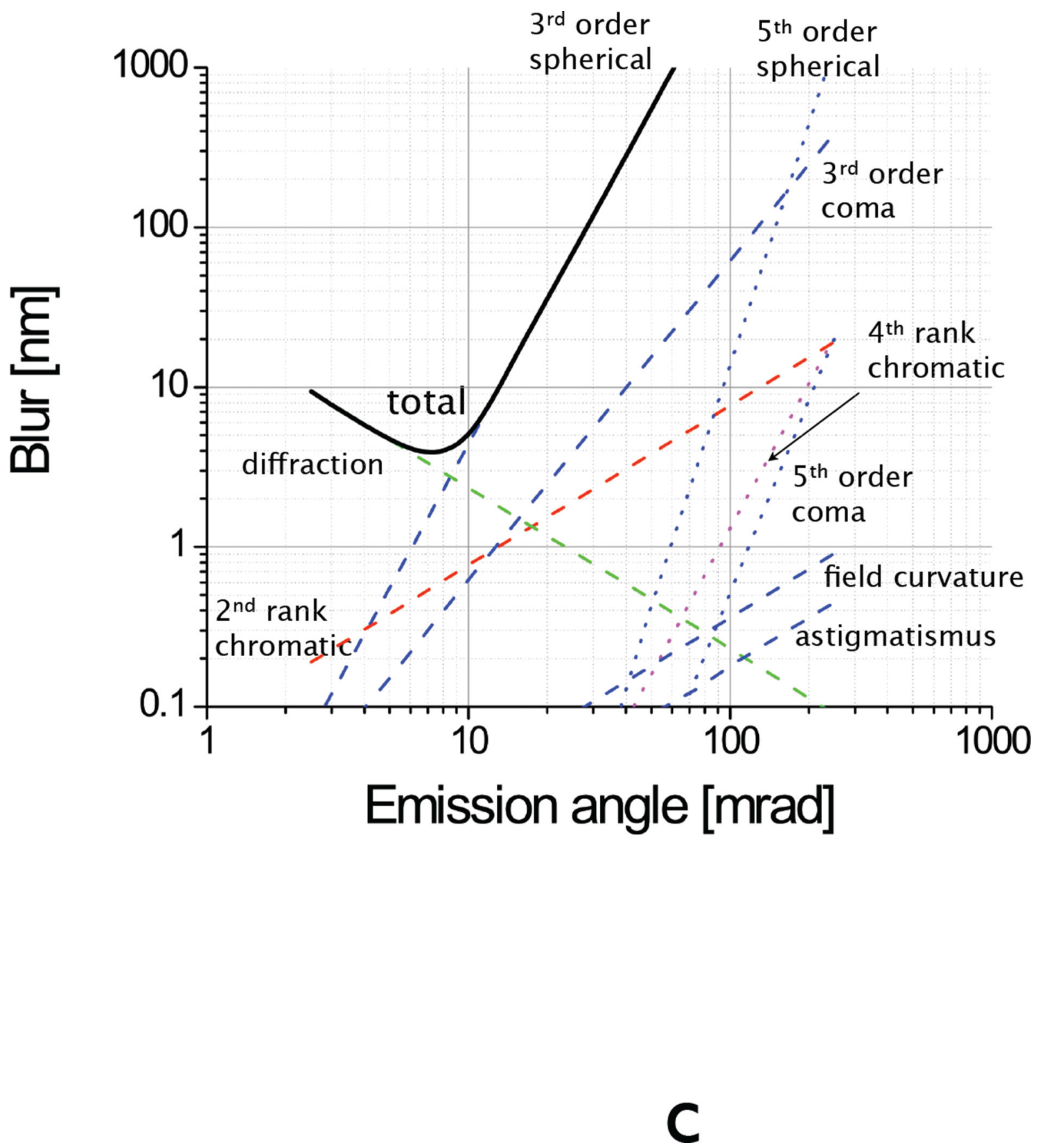

Figure 3.

Aberrations of magnetic objective lens for a field of view of $2 \mu \mathrm{m}$ and $\Delta \mathrm{E}=0.25 \mathrm{eV}$ as a function of the electron emission angle for emission energies of (a) $10 \mathrm{eV}$, (b) $100 \mathrm{eV}$ and (c) $1000 \mathrm{eV}$. 


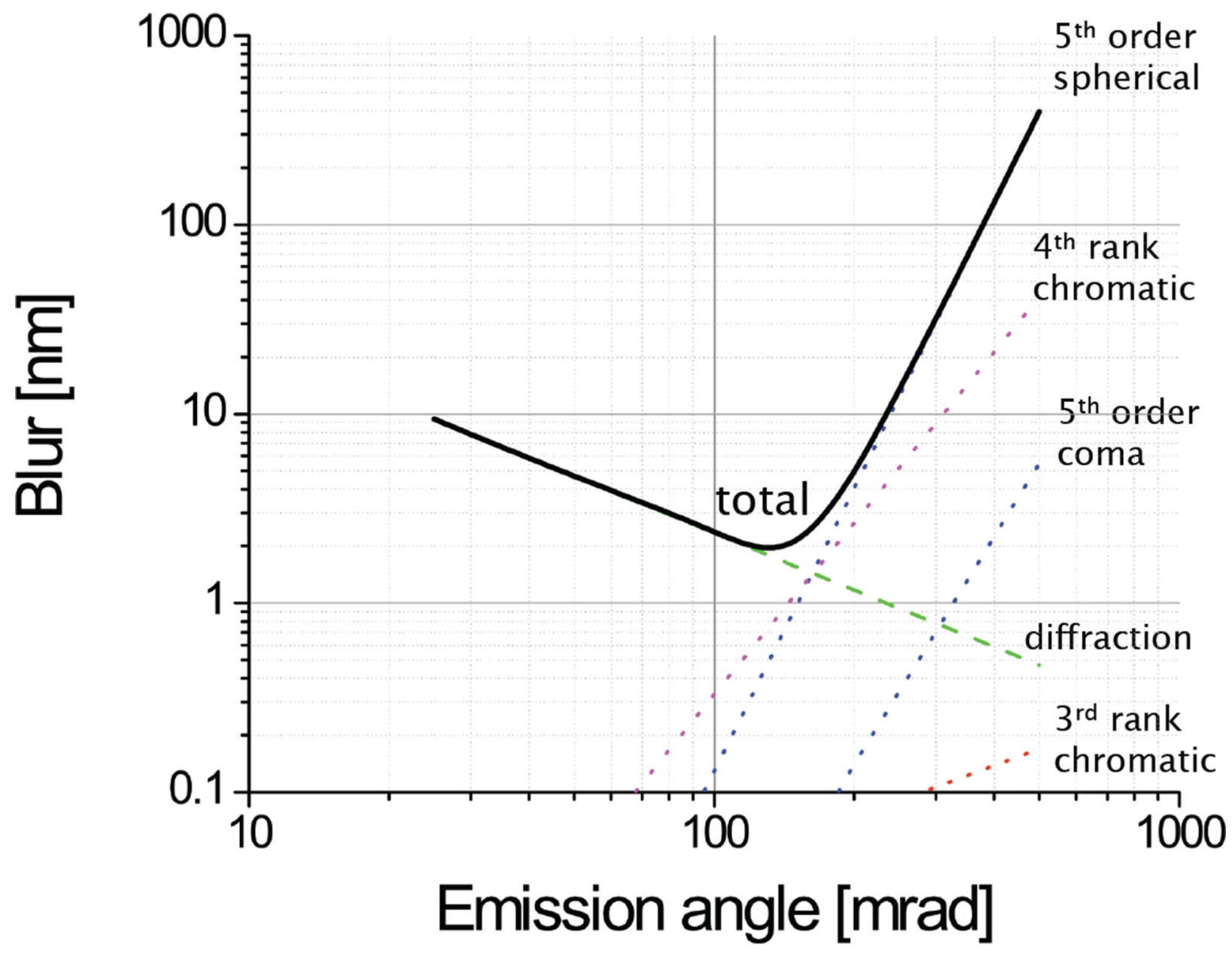

a 


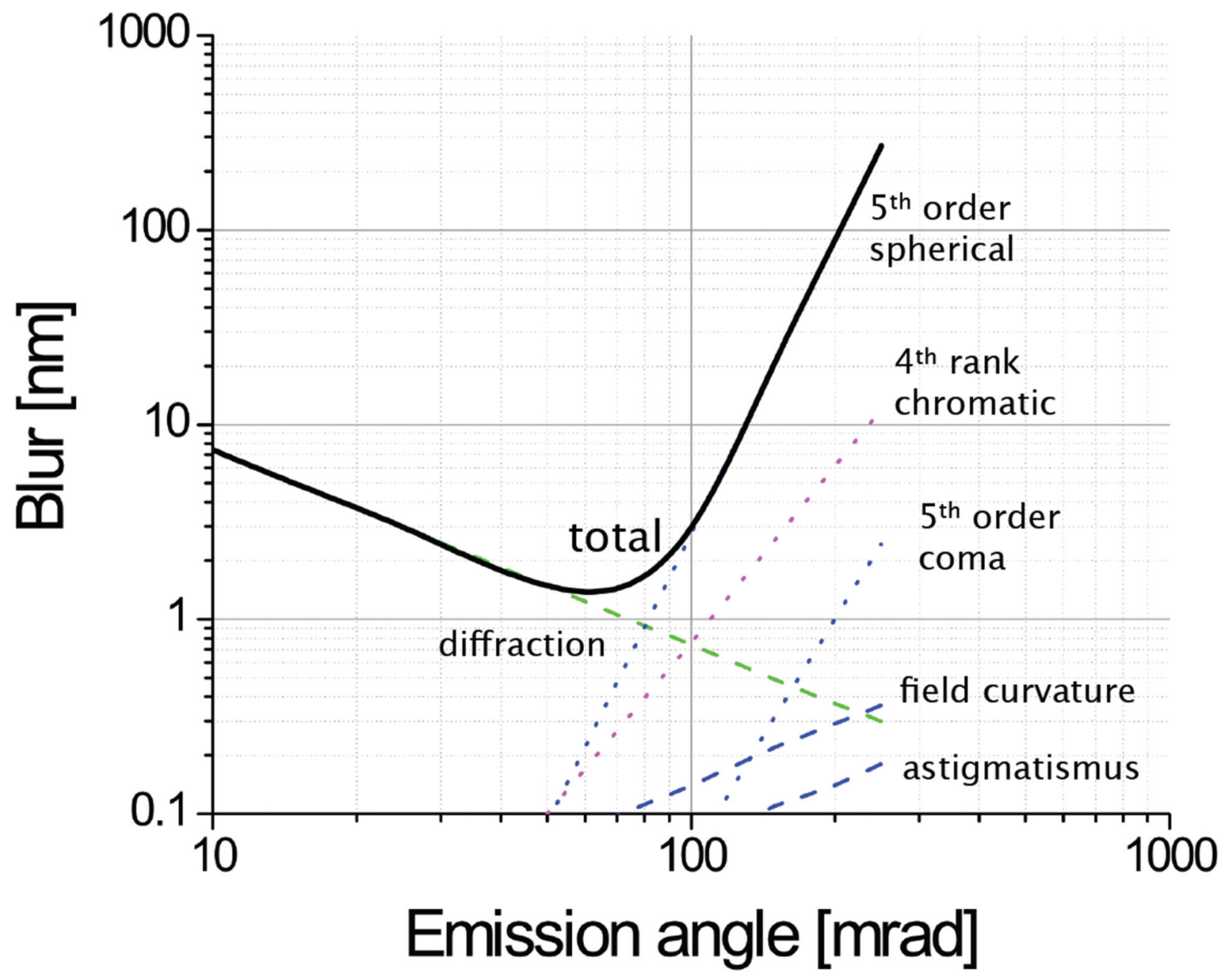




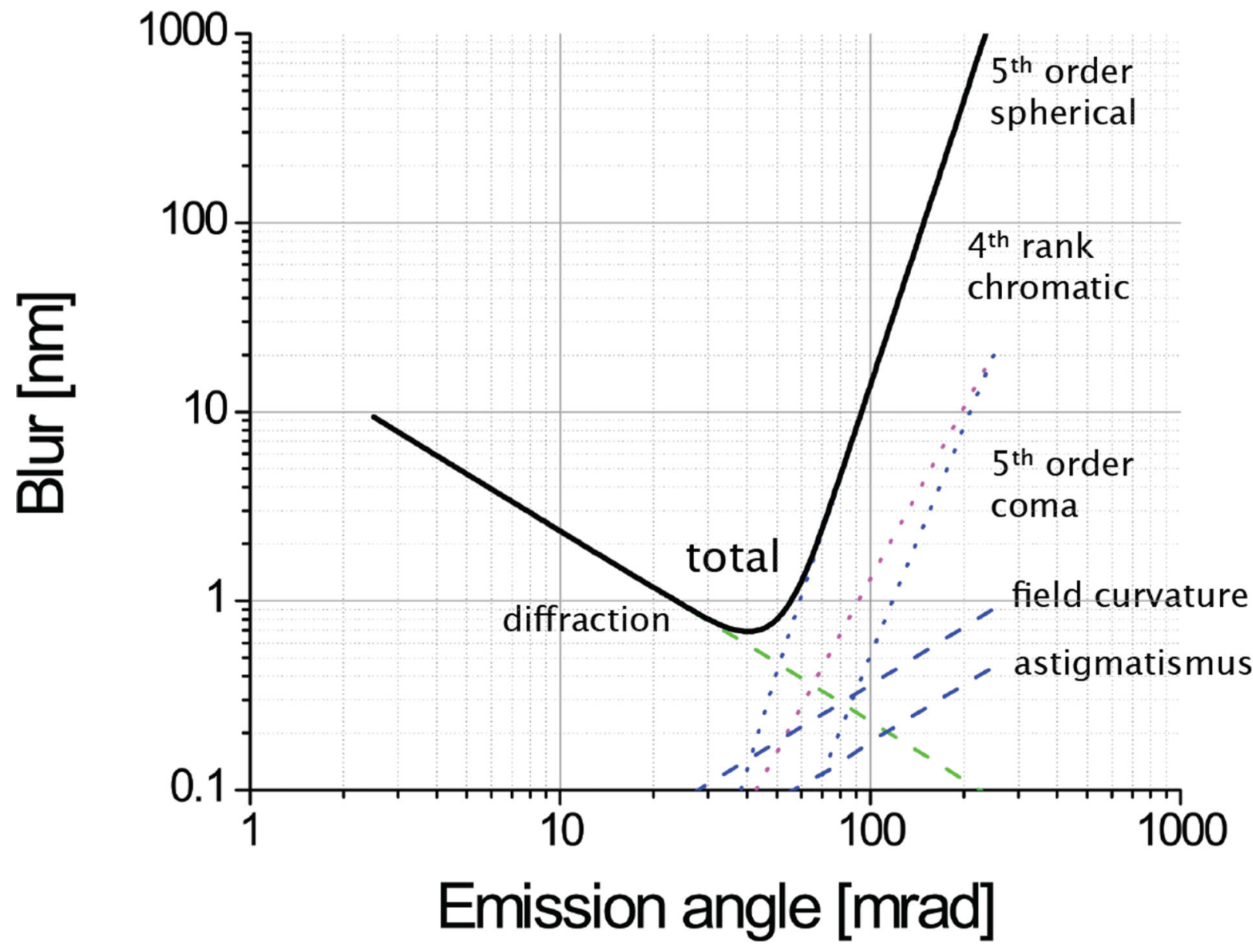

Figure 4.

Magnetic objective lens aberrations for a field of view of $2 \mu \mathrm{m}$ and $\Delta \mathrm{E}=0.25 \mathrm{eV}$ with corrected primary spherical and chromatic aberrations as function of the electron emission angle for emission energies of (a) $10 \mathrm{eV}$, (b) $100 \mathrm{eV}$ and (c) $1000 \mathrm{eV}$. 


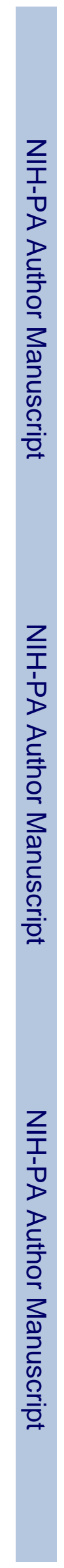

Mankos et al.

Page 32

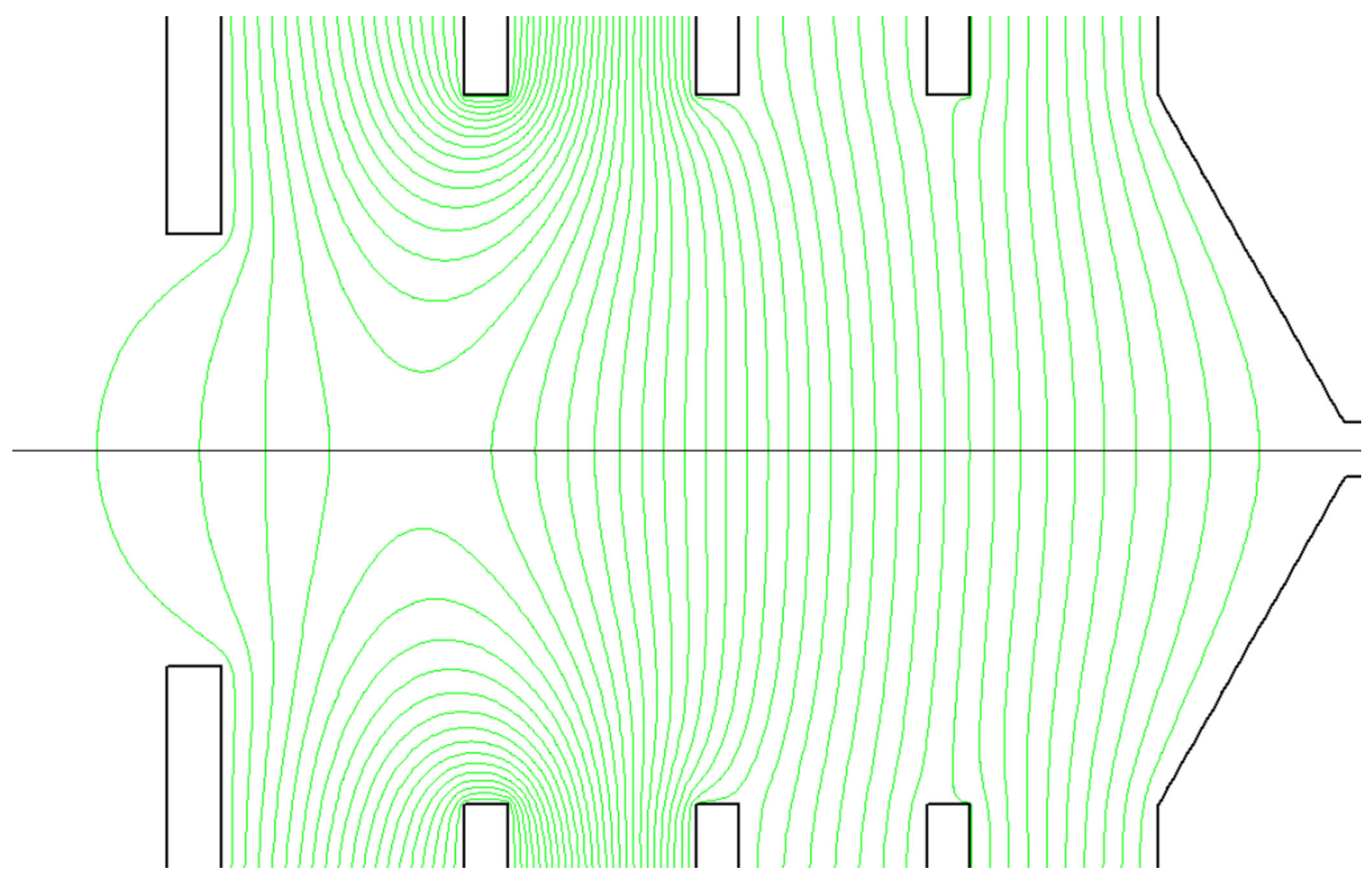

Figure 5.

Geometry and equipotential distribution of a pentode MAC.

Ultramicroscopy. Author manuscript; available in PMC 2015 October 01. 


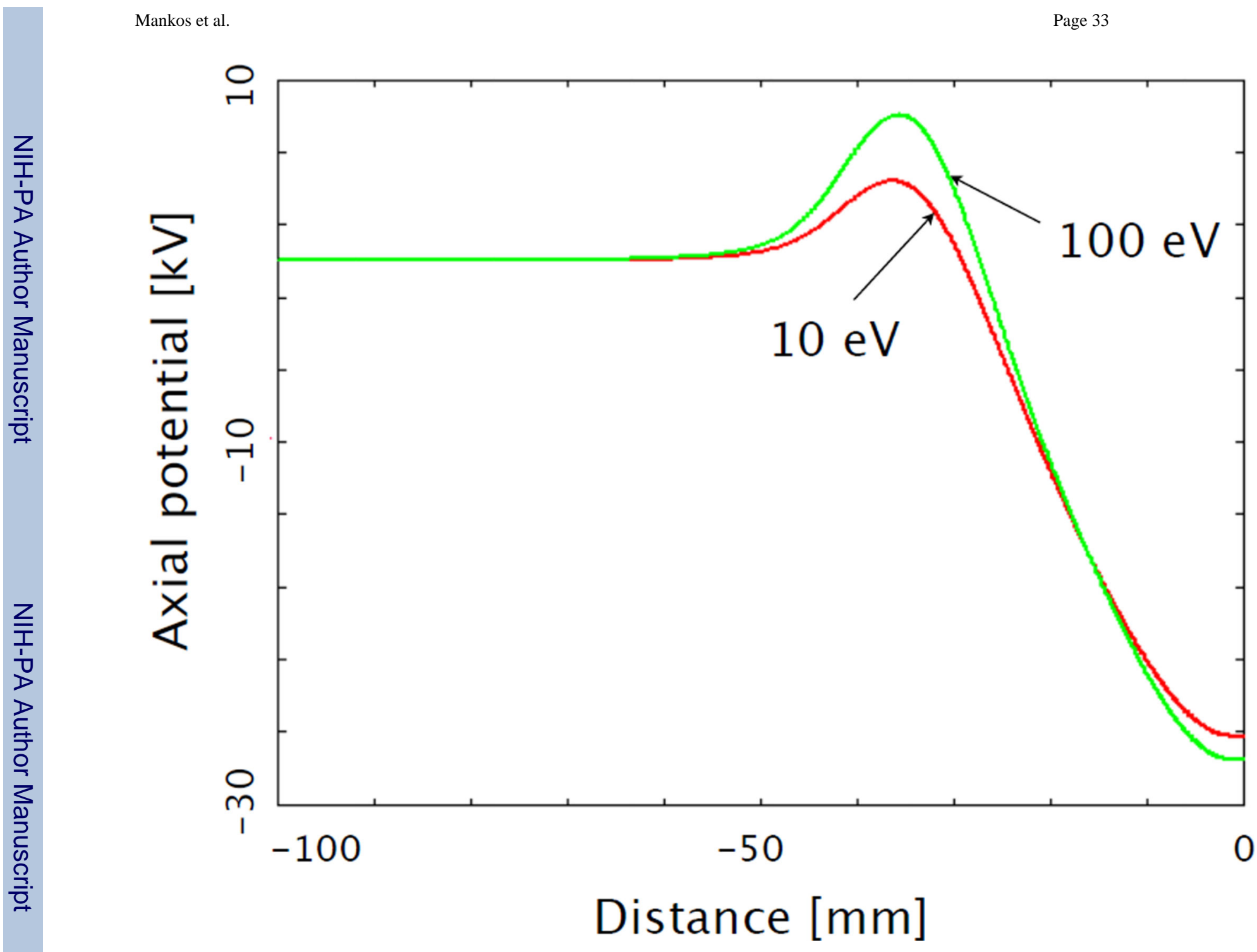

Figure 6.

Axial equipotential distribution of a pentode MAC with electrode potentials set to correct the aberrations of the objective at 10 and $100 \mathrm{eV}$. 


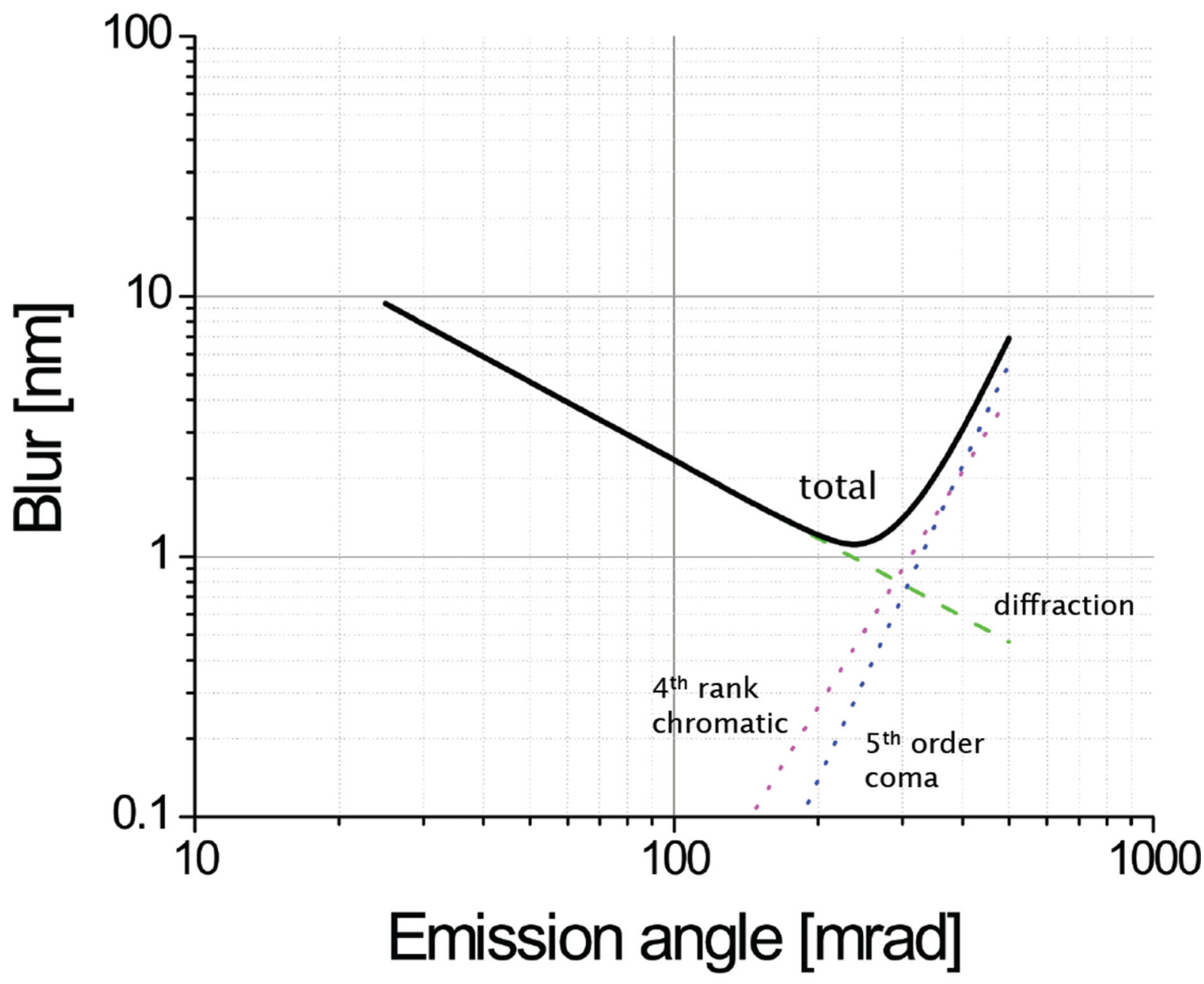

a 


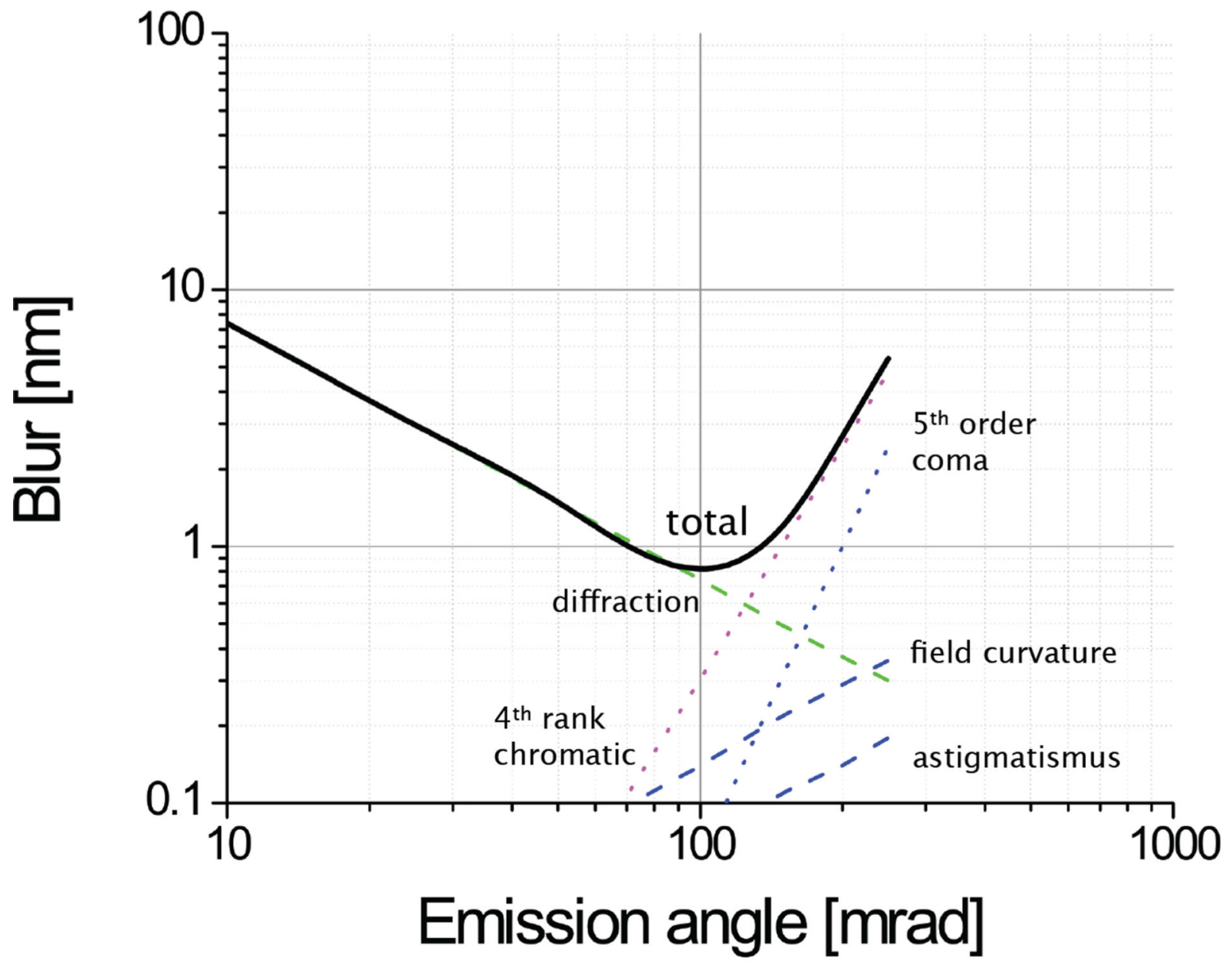

b 

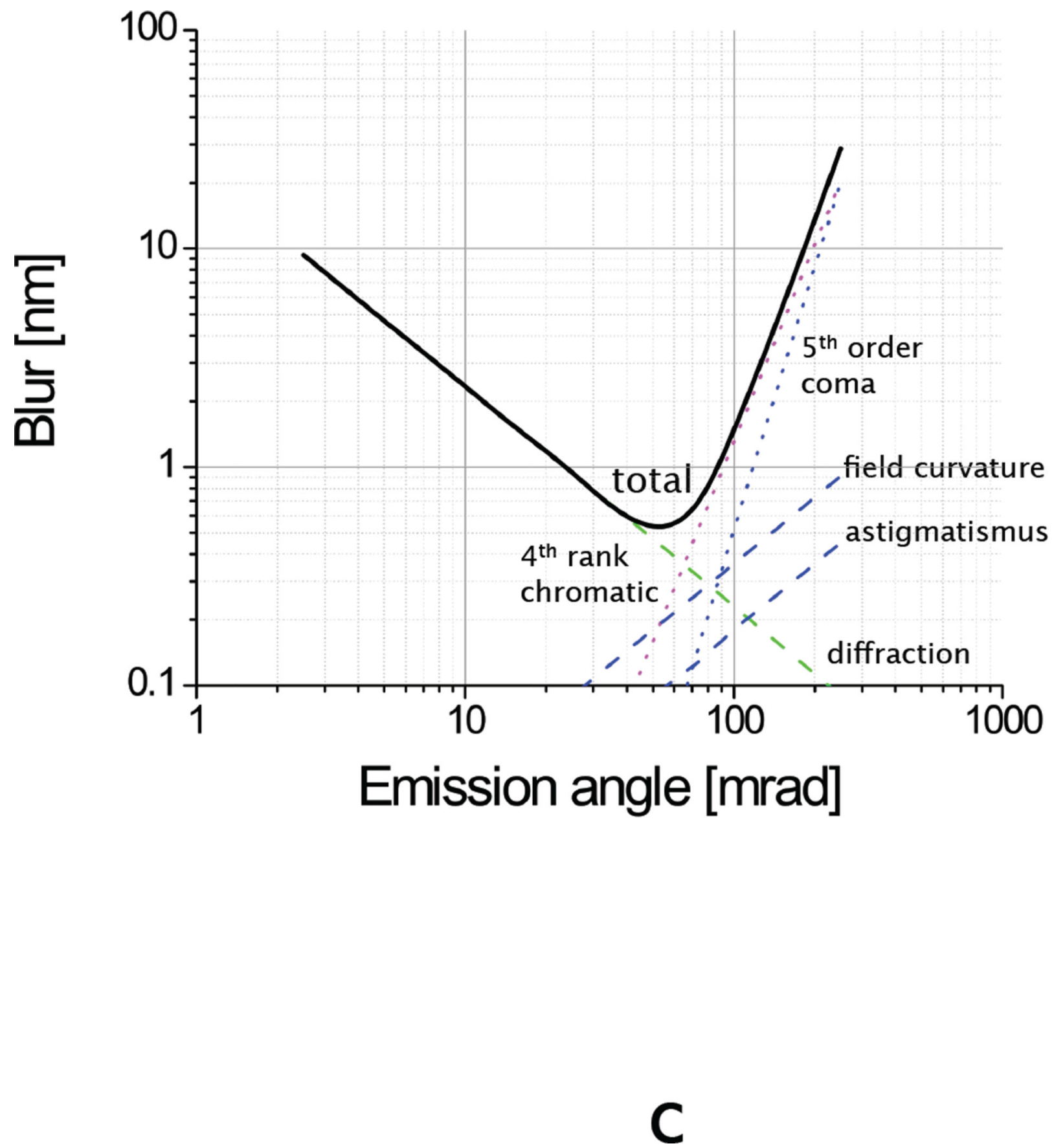

Figure 7.

Magnetic objective lens aberrations for a field of view of $2 \mu \mathrm{m}$ as a function of the electron emission angle with corrected $3^{\text {rd }}$ and $5^{\text {th }}$ order spherical and primary chromatic aberrations at (a) $10 \mathrm{eV}$ emission energy with a $25 \mathrm{meV}$ energy spread, (b) $100 \mathrm{eV}$ emission energy with a $100 \mathrm{meV}$ energy spread, and (c) $1000 \mathrm{eV}$ emission energy with a $250 \mathrm{meV}$ energy spread. 


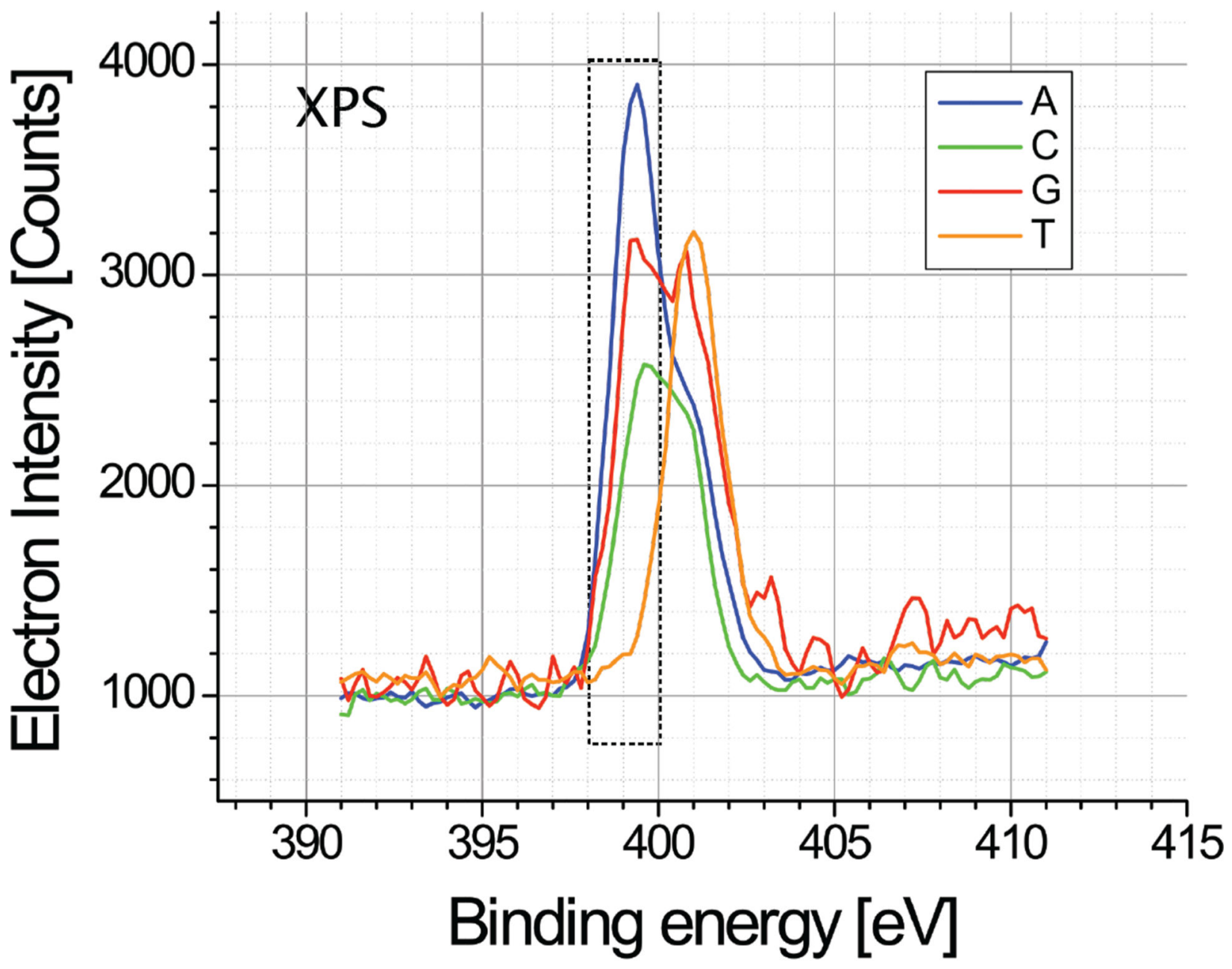

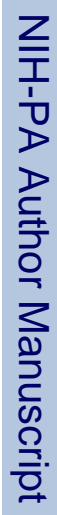

a 


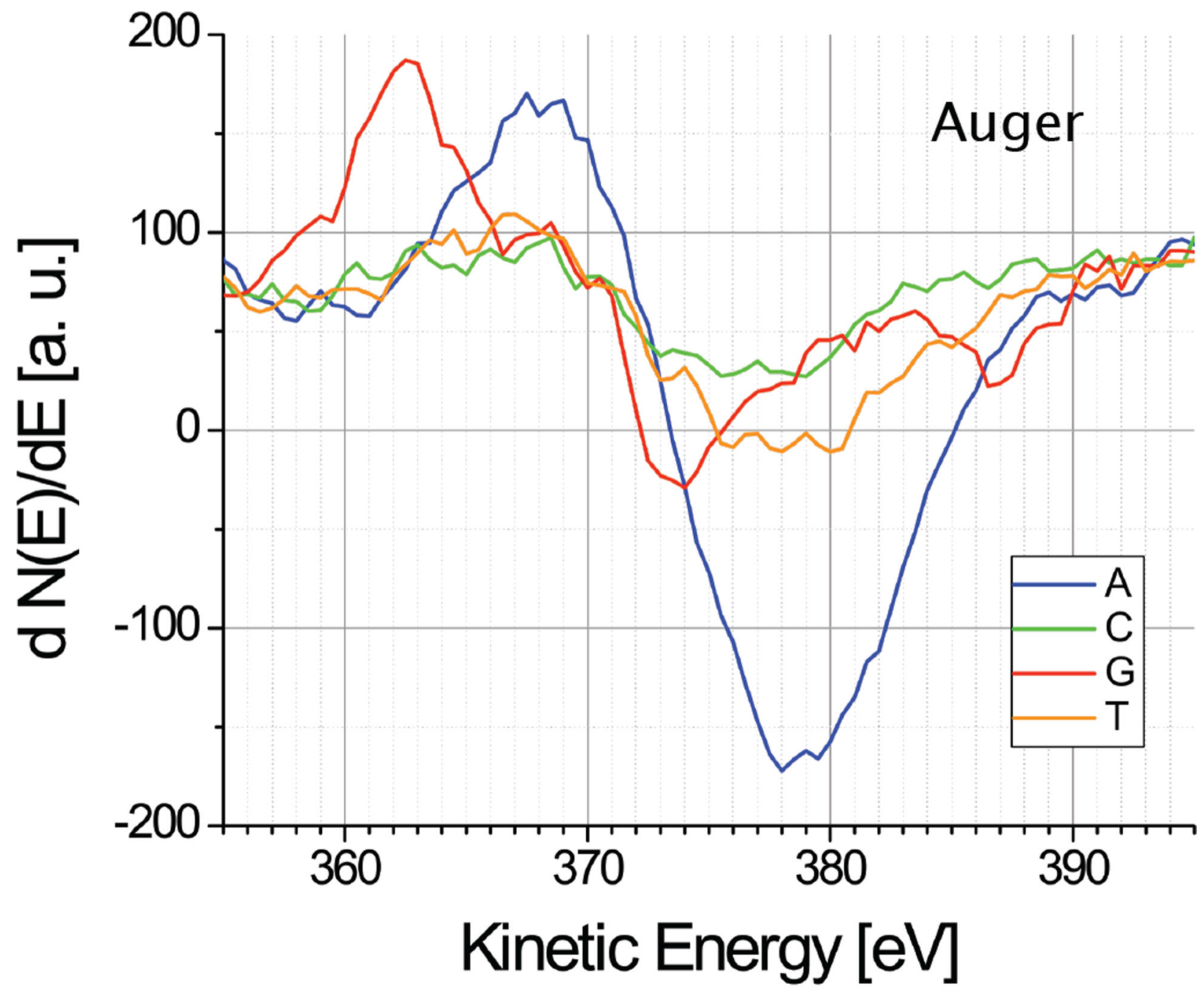

Figure 8.

XPS (a) and differentiated Auger (b) N 1s spectra for a set of four samples, prepared by immobilizing 20mers of A, C, G, and T on a Au-coated Si substrate functionalized with a monolayer of aminoundecanethiol. 
(a)

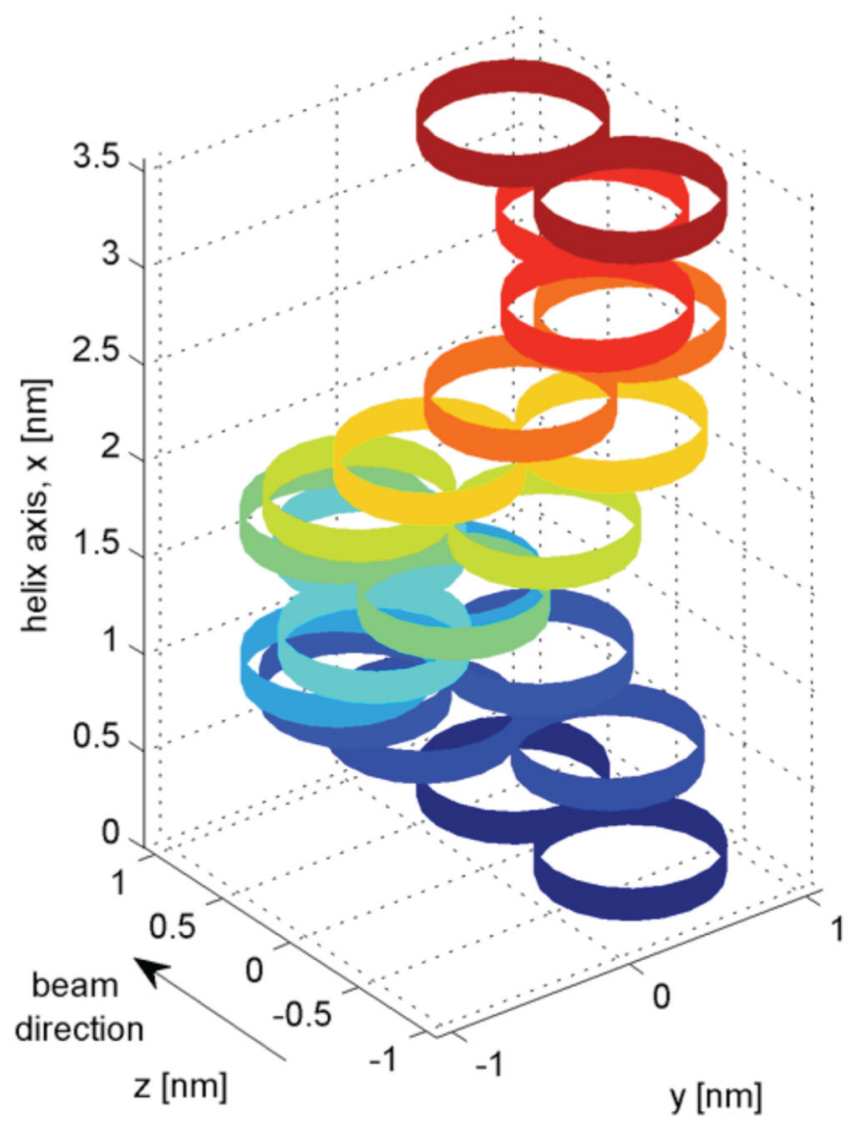

view along helix axis

(b)

Figure 9.

(a) Three-dimensional view of the model B-DNA showing eleven nucleotide pairs labeled with colors indicating their elevation along the helix axis. (b) Two-dimensional view along the helix axis of a single pair showing the region of overlap as viewed by the beam. 


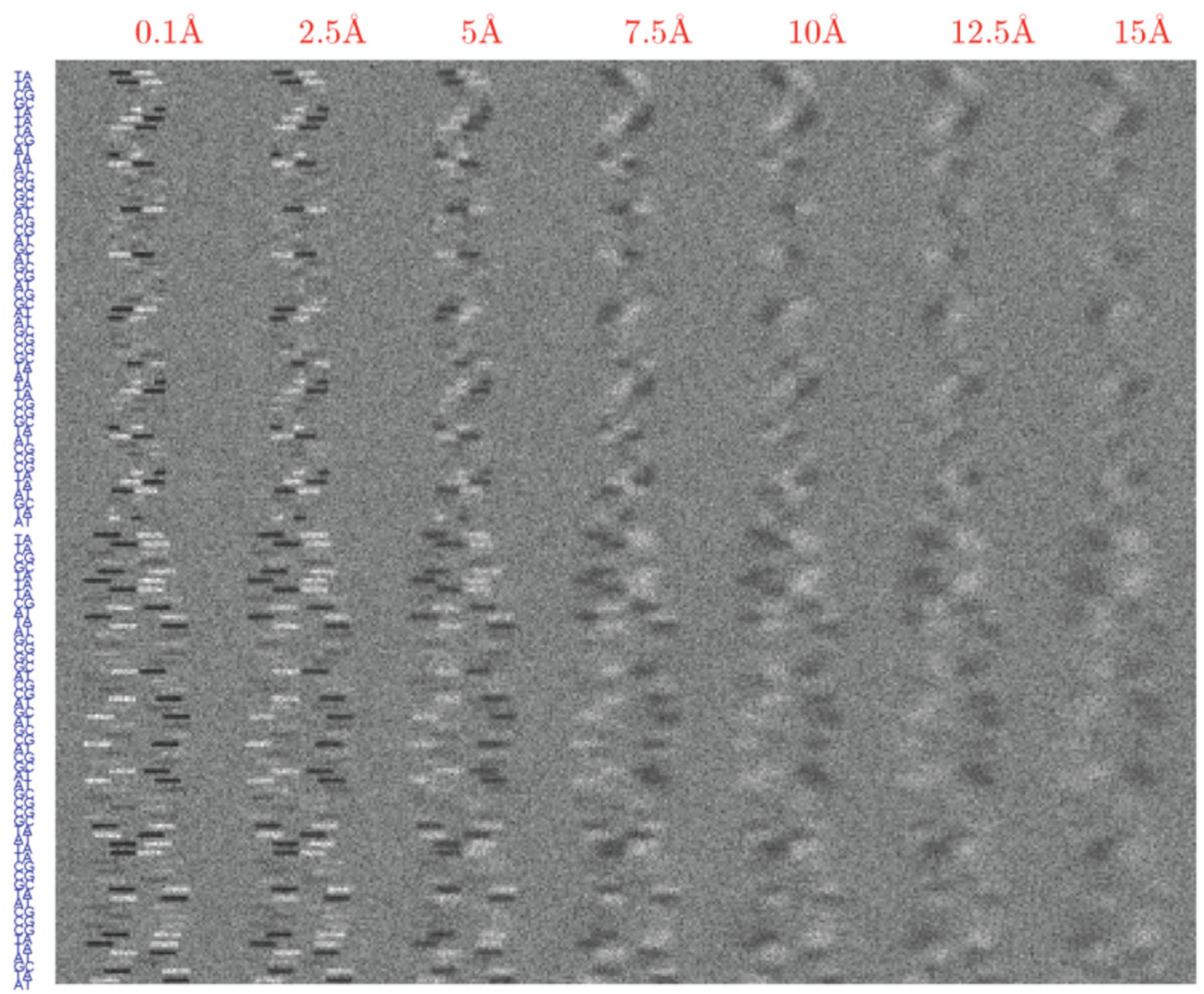

B-DNA

Figure 10.

XPS simulated images of 50 random base pairs of B-DNA and its ss-form, spliced vertically, with $1 \AA$ pixels. The sequence is identified on the left. For B-DNA, the sequence is that of the untwisted helix. Spliced from left to right are images taken with increasing optical blur as described by the PSF diameter containing $99 \%$ of the current, which is shown on top. The shot noise in the image is derived from an electron density of $100 / \AA^{2}$. Nominal intensity contrasts of 0.6 and 0.2 are used for the $A T$ and $G C$ pairs, respectively. The images are 8-bit or 256 gray-levels with [50,200] describing black and white, respectively. The substrate gray-level is set to 128 . 


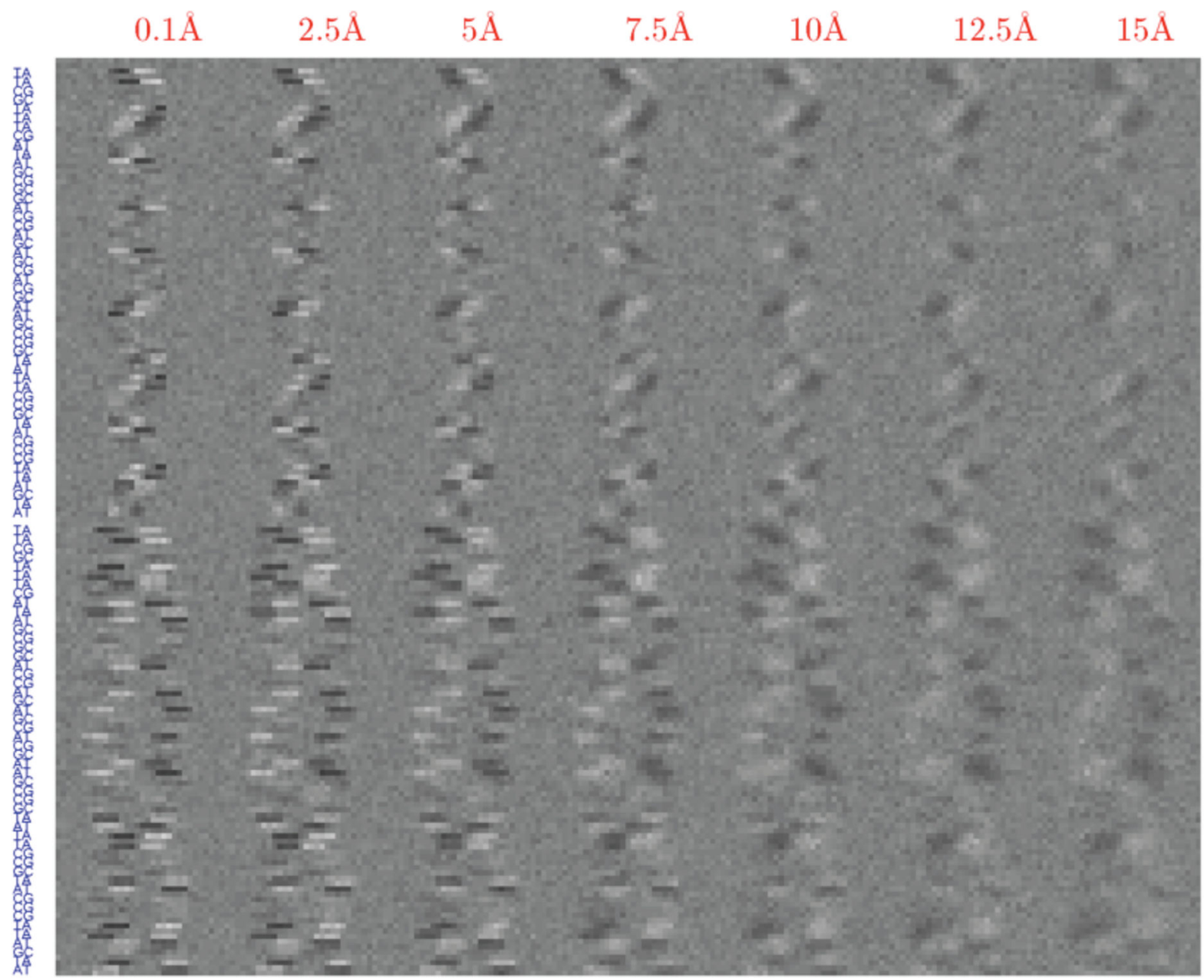

B-DNA

Figure 11.

XPS simulated images of 50 random base pairs of B-DNA and its ss-form, spliced vertically, as described in Fig. 4 with 2 A pixels. 
(a)

(b)
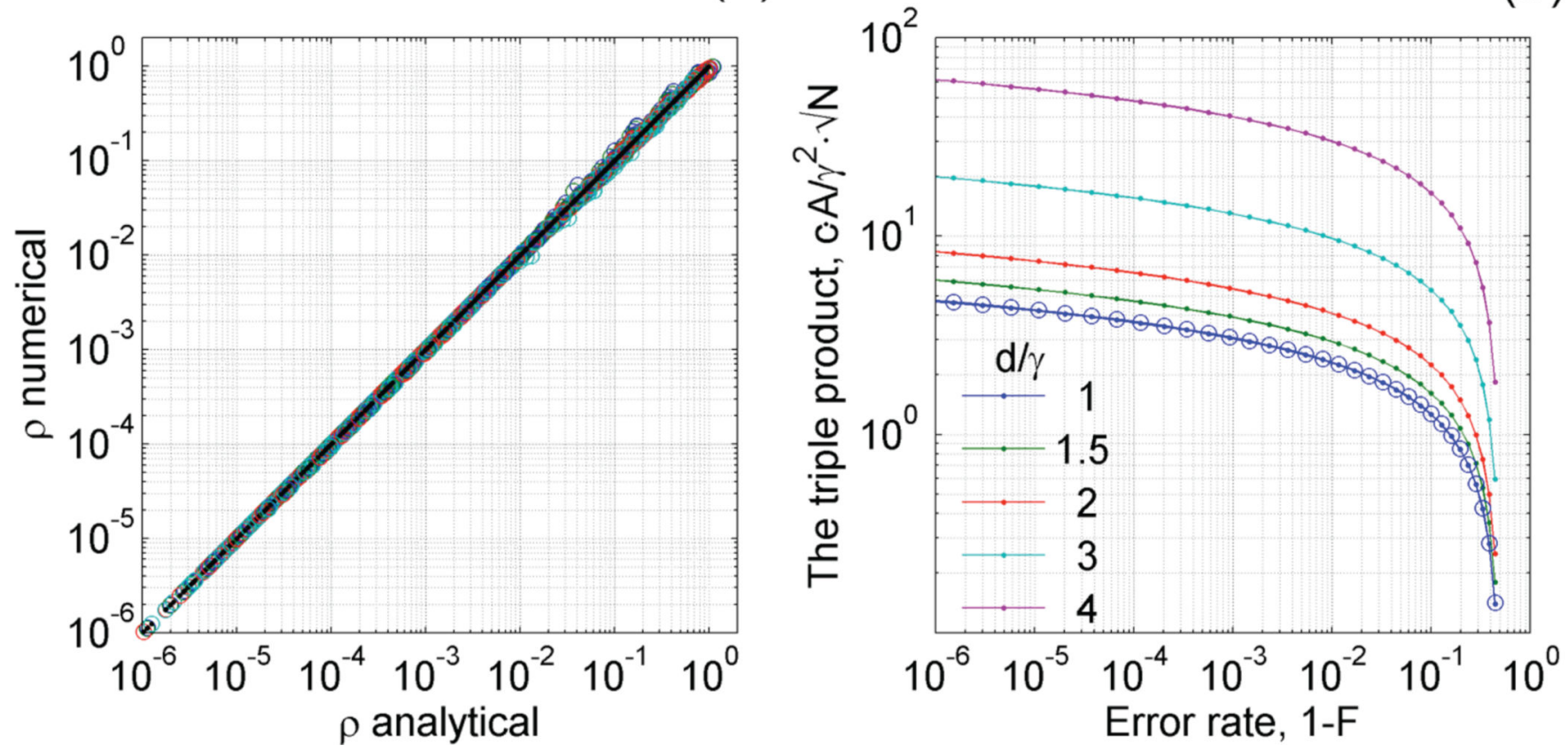

Figure 12.

(a) A comparison of the approximation for the contrast loss, $\rho$, between two alternating features separated by $\gamma$ with the numerically derived result for a range of values for the imaging and feature parameters; namely, optical blur values of $d=[1: 0.25: 8] \cdot \gamma$, pixel sizes of $p=[0.1,0.25,0.5,1.0] \cdot \gamma$, feature lengths of $l_{x}=[0.1,0.25,0.5,1.0] \cdot \gamma$, and feature widths of $l_{y}=[0.1,0.5,1.0,2.0,4.0] \cdot \gamma$. (b) The triple product of the nominal contrast, $c$, the ratio of the feature area $A$ to that of the pixel, and the square-root of the number of electrons per pixel, $N$, graphed against the error rate, $1-F$, for a few ratios of the optical blur to the feature pitch, $d / \gamma$. Here, the pixel size has been optimized to $p=\gamma$. The circles denote the result in the limit $d / \gamma \rightarrow 0$. 


\section{Table I}

Electron-optical properties of the objective lens and pentode MAC at 10 and $100 \mathrm{eV}$ electron energy.

\begin{tabular}{|c|c|c|c|c|}
\hline \multirow[b]{2}{*}{ Parameter } & \multicolumn{2}{|l|}{ Objective lens } & \multicolumn{2}{|l|}{ Pentode MAC } \\
\hline & $10 \mathrm{eV}$ & $100 \mathrm{eV}$ & $10 \mathrm{eV}$ & $100 \mathrm{eV}$ \\
\hline Magnification & 7.93 & 8.20 & 1.00 & 1.00 \\
\hline $3^{\text {rd }}$ order spherical aberration coeff. $[\mathrm{m}]$ & $3,150.0$ & $2,739.5$ & -3153.1 & -2739.9 \\
\hline $2^{\text {rd }}$ rank chromatic aberration coeff. [m] & -11.805 & -4.508 & 11.744 & 4. 498 \\
\hline $5^{\text {th }}$ order spherical aberration coeff. $[\mathrm{m}]$ & $-5.607 \times 10^{8}$ & $-4.776 \times 10^{7}$ & $-5.637 \times 10^{8}$ & $-4.751 \times 10^{7}$ \\
\hline $3^{\text {rd }}$ rank chromatic aberration coeff. [m] & -6252 & -255 & 12.55 & 22.8 \\
\hline $4^{\text {th }}$ rank chromatic aberration coeff. $[\mathrm{m}]$ & $9.371 \times 10^{6}$ & $7.837 \times 10^{5}$ & $-1.921 \times 10^{5}$ & $-1.603 \times 10^{5}$ \\
\hline
\end{tabular}

